\title{
CORRELATION OF MEDIAL THALAMIC NUCLEAR ACTIVITY WITH CORTICAL AND SUBCORTICAL NEURONAL ARCS
}

MARY JANE C. SHOWERS ${ }^{1}$

Laboratory of Comparative Neurology, Department of Anatomy, The University of Michigan, Ann Arbor, Michigan"

SIX FIGURES

This study is concerned with certain functions of the medial nucleus of the dorsal thalamus and the way in which these are reflected by its various connections within the nervous system. This work tends to emphasize that this nucleus is a part of a complex motor and sensory system involving connections with the central nervous system at hemisphere and brain stem levels. For documentation of this statement the results of stimulation and ablation experiments are reported and correlated with the underlying anatomical relations as revealed by a study of the brains of the animals operated upon in these experiments.

\section{METHODS AND MATERIALS}

The microscopic anatomy of the brain of Macaca mulatta was investigated in order to establish the normal topography of the areas involved experimentally. The material studied was from the Huber Neurological Collection. A normal series of sections of the brain had been stained by the Weil technique and a companion series stained with toluidin blue. These provided a correlative comparison of nuclei and fiber tracts in the various parts of the brain.

\footnotetext{
${ }^{1}$ This paper is based on a thesis submitted in partial fulfillment of the requirements for the degree of Doctor of Philosophy in the University of Michigan.

"The work was assisted by grants from the Walter C. Hill Fund and animals provided by the Parke, Davis Pharmaceutical Company for which the writer wishes to express appreciation.
} 
The slides resulting from the experimental work were prepared by the Swank-Davenport ('35) modification of the Marchi method. This reveals degenerating myelinated fibers. Experimental animals were sacrificed at the termination of a two to 4 week period extending from the completion of the experimentation. Under ether anesthesia the thorax was opened and the aorta cannulated through the left ventricle. Perfusion of the arterial system with approximately $1000 \mathrm{ml}$ of $10 \%$ formalin was carried out. The brain was removed and placed in $10 \%$ formalin to complete its fixation. Examination of the resulting slides showed a consistent distribution of two forms of artefact similar to those illustrated by Smith ('56a, '56b). Pseudo-Marchi dust of very fine granules was seen diffused throughout the cortical areas. Pseudo-Marchi staining of fibers was evident in the optic chiasm, optic tracts, lateral geniculate nucleus, oculomotor nerve roots, substantia nigra, and posterior commissure.

Observation and testing of the experimental animals included an evaluation of the following items preoperatively and postoperatively. Cardinal signs of temperature, pulse, and respiration were recorded. The conscious level was assayed in descriptive terms of comatose, drowsy, lethargic, active, excited, attentive, and tractable. Sensory modalities relevant to deep and superficial pain, deep and superficial touch, sight, hearing, and taste were listed and the results noted. Motor activity was checked in regard to tone, paresis, placing, tics, chorea, circus movements, eye movements, vestibular responses, tendon reflexes, pupillary reflexes, and Babinski, grasp, Hoffman and Rossilimo reflexes. Sweating and shivering, eating habits and elimination were observed. The animal's behavior toward the examiner was judged.

In the experimental procedure either a preoperative medication of atropine sulfate $(0.04 \mathrm{mg})$ was given subcutaneously and ether anesthesia employed, or a solution of morphine sulfate $(1 \mathrm{mg})$ and scopolamine hydrobromide $(0.04 \mathrm{mg})$ was injected hypodermically antecedant to intravenously administered sodium pentothal in a $2 \frac{1}{2} \%$ to $5 \%$ solution. After 
induction of anesthesia to the stage of loss of consciousness and muscular relaxation, the head of the animal was prepared by shaving and then cleansing with soap, water, alcohol, and zephiran solution. The skull openings were made with dental burrs and enlarged with a rongeur. Dural, muscular, subcutaneous, and skin repairs were made with nylon thread. The bone defect was not repaired. Bleeding was controlled by ligation, electrocoagulation, and application of gelfoam. A small cotton sponge sutured to the skin and covered with collodian was used to protect the operative incision.

When cortical ablation was planned, the surface to be studied was destroyed by gross dissection with manual and electrocauterizing scalpels. Cotton pledgets and suction were used to remove the debris. When stereotaxic lesions were planned, Labtronics Stereotaxic Instrument (model 4) was used to orient a monopolar electrode. This electrode was a $5 \mathrm{~cm}$ steel needle fixed in hollow steel tubing $9.5 \mathrm{~cm}$ long and $1.5 \mathrm{~mm}$ wide. The electrode was insulated with lacquer up to its tip. A metal rectal plug served as the indifferent electrode. Using a Grass Stimulator (model 3C), direct current of 300 mamp for $60 \mathrm{sec}$ or $3 \mathrm{ma}$ for $15 \mathrm{sec}$ was passed through the electrode to produce a lesion.

Prior to all stereotaxic lesions, the area of the brain involved in the experiment was stimulated. A monopolar electrode arrangement as previously described was used most of the time. Square wave impulses with a frequency of 40 per sec, a duration of $1 \mathrm{msec}$, an intensity of 1 to $14 \mathrm{v}$, constituted the usual type of stimulation. Minimal and maximal stimulus strengths for a specific motor activity were determined. They varied considerably among the animals used. In several instances, stimulation was carried out with a concentric electrode. Fine-gauge steel tubing $0.5 \mathrm{~mm}$ diameter and $11 \mathrm{~cm}$ long enclosed an insulated copper wire. The copper wire leading from the electrode was shielded by polyethylene tubing and terminated in banana plugs. When cortical stimulation was performed, a blunt-tipped silver wire insulated with plastic tubing served as the electrode. 
RESULTS OF EXPERIMENTATION

Protocols 1, 4, 8, 10, 10a and 106

Several animals in which the essential procedure was the stimulation and the destruction of the medial nucleus of the thalamus will be considered together. Animal 1, a female Macaca mulatta, approximately $9 \mathrm{lb}$ in weight, was operated upon three times at biweckly intervals $(10 / 21 / 55,11 / 2 / 55$ and 11/16/55). A monopolar electrode oriented by stereotaxic aid was used to stimulate and destroy the medial nucleus of the thalamus. Following the sequence suggested by the dates above, the sites stimulated were rostral to caudal in 4 positions in the left diencephalon, caudal to rostral in 4 positions in the right diencephalon, and rostral to caudal in 8 positions in the left diencephalon.

Animal 4, a male Macaca mulatta, approximately $12 \mathrm{lb}$ in weight, was operated upon three times at biweekly intervals $(2 / 14 / 56,2 / 28 / 56$ and $3 / 13 / 56)$. A monopolar electrode, oriented by stereotaxic aid was used to stimulate and later destroy the medial nucleus of the thalamus. In the sequence indicated by the dates above, the sites stimulated proceeded from rostral to caudal in 4 positions and caudal to rostral in 4 positions in the left diencephalon, rostral to caudal in 5 positions and caudal to rostral in 5 positions in the left diencephalon, and rostral to caudal in 5 positions and caudal to rostral in 5 positions in the right diencephalon.

Animal 8, a male Macaca mulatta, approximately $5 \mathrm{lb}$ in weight, was operated upon twice at a week's interval $(6 / 29 / 56$ and $7 / 1 / 56)$. During the first surgical procedure, a monopolar electrode, oriented by stereotaxic aid, supramaximal stimulation was used to stimulate the left medial nucleus of the thalamus at 4 positions from rostral to caudal and at 4 positions caudal to rostral, until convulsive movements occurred with each position stimulated. Using similar apparatus a second time, the right medial nucleus of the thalamus was stimulated and destroyed at 4 positions, rostral to caudal. By directing the electrode across the midline, the left medial nucleus was also stimulated and destroyed at three positions. 
Animal 10, a male Macaca mulatta, approximately $4 \mathrm{lbs}$ in weight, was operated upon twice at a two-week interval $(7 / 20 / 56$ and $8 / 3 / 56)$. During the first procedure a subpial ablation of area 22 in the left superior temporal gyrus was carried out by blunt dissection with a cotton sponge. During the second surgical procedure, the left medial nucleus of the thalamus was stimulated at 4 positions, from rostral to caudal. An unsuccessful attempt was made to cross the midline in order to stimulate and destroy the right medial nucleus at 4 positions.

Animal 10a, a female Macaca mulatta, approximately $7 \mathrm{lb}$ in weight, was operated upon on $1 / 15 / 57$. The right medial thalamic nucleus was stimulated and destroyed at 9 positions rostral to caudal and at 4 positions caudal to rostral. Animal $10 \mathrm{~b}$, a female Macaca mulatta with a weight about $6 \mathrm{lb}$ was operated upon on $2 / 12 / 57$ and $2 / 19 / 57$. The procedure described for animal 10 was duplicated. Five positions rostral to caudal and 5 positions caudal to rostral were explored in the right diencephalon.

The pattern of reaction in all of these animals was the same. When the rostral parts of the nucleus were stimulated, movements occurred in the contralateral lower extremity (fig. 1, A-1 and A-2). These movements were flexion, in a clonic fashion, of the toes, the ankle, the knee and the hip. As the stimulating electrode was placed more caudally, clonic movements could be observed in the trunk musculature. Change in respiration also occurred at this point. It was usual for the respiratory excursions to increase in frequency, then become slow and stertorous, and then cease. Still farther caudalward, upper extremity movements (fig. 1, B-1 and B-2) on the contralateral side were elicited in the sequence observed for lower extremity; namely, the fingers, the elbow, and the shoulder joints were clonically flexed. At the most caudal position of stimulation, the contralateral face musculature alternately contracted and relaxed (fig. 1, C-1 and C-2). It was not uncommon, at this point in stimulation, to observe that the tongue was protracted and retracted. The movements 
described were obtained first when the stimulation was in a 2 to $5 \mathrm{v}$ range. As the strength of the stimulation exceeded $5 \mathrm{v}$, it was possible to bring other parts of this pattern into play, successively or simultaneously, until a generalized convulsion ensued when the stimulus strength ranged between $10 \mathrm{v}$ and $14 \mathrm{v}$. When the stimulating electrode was moved medially, facial movements predominated. If the stimulating electrode was shifted laterally, movements of the lower extremity predominated. Movements involving the upper extremity and trunk musculature could be found between the medial and lateral parameters of stimulation.

Immediately following surgery, most of these animals shivered violently for 20 to 30 minutes and felt cold to touch. In 5 instances (and 4 different animals), where the rectal temperature was checked before and after the surgical procedure, there were drops of body temperature from $1.2^{\circ}$ to $2.3^{\circ} \mathrm{C}$. Random comparison of the protocols of three other animals having cortical ablations showed a range of $0.1^{\circ}$ increase to $1.3^{\circ}$ decrease in rectal temperature.

In all cases there was a lowered activity level of these animals following ablation of the medial nucleus. All of the animals were more tractable and easier to handle following: the procedure. All of them had a tendency to assume a pose in which the head was hyperextended as the animal sat crouched in the corner (fig. 1F). The attitude resembled one of day-dreaming. It was possible to take pictures of animal 8 (fig. 1E) with the cage door open while in this posture. Along with the pose, animal 4 smacked his lips, ground his teeth, and yawned frequently. Three of the 6 animals demonstrated a very fine tremor at rest. The movements were present in muscles of the hand and foot contralateral to the ablated medial nucleus. Animal 10 had a marked deviation of the head, the neck, and the eyes to the right (fig. 1F). Because of this, the monkey was dysmetric in placing and jumping movements, both overshooting and undershooting the intended mark. Animals 1 and 10a showed a marked inerease in water intake postoperatively, but animal 
4 manifested the converse behavior. The other animals included in this protocol consumed more water postoperatively than before the experiments. The water pans holding approximately $500 \mathrm{~cm}^{3}$ were checked twice a day.

Necropsy of these animals evidenced several small electrode marks on either side of the central fissure about $3 \mathrm{~mm}$ from the midline. A study of the Marchi slides indicated that the electrode positions produced lesions in the medial nucleus in all of the animals. There were lesions in structures through which the electrodes passed to reach the medial nucleus (fig. $3 A$ ). Among such structures were areas 3 and 4 , the cingulate gyrus, the fornix, and the anterior nuclear group of the dorsal thalamus. In animal 6, one electrode position was in the internal medullary lamina and the nucleus ventralis lateralis. In animal 10, one electrode position extended below the medial nucleus into the centromedian nucleus and the red nucleus of the midbrain and another electrode mark abutted the internal medullary lamina and the nucleus ventralis posterior. In animals 10a and 10b, 4 lesions were evident in the internal medullary lamina and the nucleus ventralis lateralis.

Degeneration granules from very finely medullated fibers collect rostral and medial to the medial nucleus and extend downward in the diencephalic periventricular system to the midline thalamic nuclei and the hypothalamic nuclei. The position is indicated at DPS, figure 3A. The latter include the paraventricular nucleus, the ventromedial nucleus, the dorsomedial nucleus, and the lateral hypothalamic area. Fascicles that are degenerating are aggregated rostral and lateral to the medial nucleus and pass over the internal medullary lamina (fig. 3A at IML), then over the nucleus ventralis lateralis, and through the reticular nucleus and the external medullary lamina (fig. 3A at EML) to enter the anterior thalamic radiations in the anterior limb of the internal capsule. A few of these fibers appear to extend into the caudate nucleus and the lentiform nucleus, but the remainder continue in the anterior limb to the orbitofrontal cortex (fig. 5B; see also Fulton, '49). Other degenerating fascicles reach the basal 
ganglia by passing through the nucleus ventralis lateralis (fig. 3A at NVL and the internal capsule directly, or to the thalamic fasciculus and then across the posterior limb of the internal capsule to globus pallidus. Some of these last appear to continue across the ansa lenticularis and the zona incerta to the subthalamic nucleus and, by the inferior thalamic peduncle, to the orbital gyri of the frontal lobe. The converse of this conduction is described in protocol 5 and shown at ITP, figure 4C. There is diffuse degeneration in superior, middle, and inferior frontal convolutions and superior, middle and inferior temporal gyri that cannot be related unequivocally to the thalamic lesions.

\section{Protocol 2}

A female Macaca mulatta, weighing approximately $4 \mathrm{lb}$, was operated upon on $11 / 9 / 55$. After a craniectomy, the cortical points were stimulated first in a clockwise direction and caudal to the inferior ramus of the arcuate fissure and then counterclockwise and rostral to the inferior ramus of this fissure. Movements involving flexion of the right upper extremity, the right side of the face and the left side of the face, and conjugate deviation of the eyes toward the left were observed with this sequence of stimulation. A rostral to caudal stimulation of the inferior frontal convolution elicited movements of the right side of the face. The cortex above and below the principal fissure (fig. $5 \mathrm{~A}, 45,46,8 \mathrm{~A}, 8 \mathrm{~B}$ ) was ablated.

Immediately postoperatively, this monkey felt cold to touch. She showed a transient vertical nystagmus and left conjugate deviation of the eyes. The left lower extremity was held in marked flexion at hip, knee, ankle, metatarsal-phalangeal, and interphalangeal articulations. The right lower extremity was extended at the corresponding joints. There was hypertonicity of the limb musculature. The tonicity, the posture and the temperature returned to their preoperative status in the three weeks during which the animal was under observation. This animal was easily frightened. Circus move- 
ments by the monkey were almost continual and counterclockwise during its waking span.

The cortex of the right side was exposed on 11/30/55 under surgical aseptic technique. The position in the cortex from which the contralateral leg movements were obtainable from area 5 of the parietal lobe (fig. $5 \mathrm{~A}, 5$ ) was determined by surface stimulation. Area 5 was then ablated. The depression of respiratory rate apparent during the procedure did not persist. Immediately following the reaction to anesthesia, both lower extremities showed increased tonicity and were held in flexion at hip, knee, ankle, metatarsal-phalangeal, and interphalangeal articulations. During the subsequent two weeks, the circus movements recurred. The animal was less fearful and would accept food from the examiner. There were occasional high pitched, sharp outcries which had no apparent concomitant association.

Necropsy indicated destruction of a region of the left middle and the left inferior frontal convolutions including areas 8 , 46 , 45, and 12 (fig. $5 \mathrm{~A}$ and $5 \mathrm{~B}$ ). A small portion of area 5 in the superior parietal convolution of the right side had been ablated. A study of the microscopic material confirmed the extent of the lesions established by gross inspection. There was no degeneration of fibers rostral to the level of the frontal lobe lesion. Degenerated fascicles pass from area 45 to areas 46 and 12 of the same side and from area 8 to the superior frontal convolution of the same side. Degenerating fibers also extend around the anterior horn of each ventricle after having passed through the genu and rostrum of the corpus callosum. There are fibers from areas 45 and 12 to the contralateral hemisphere via the body of the corpus callosum. These are especially numerous to the right frontal operculum. Thus, right association paths of short fibers connect the cortical gyri above and below the principal fissure. These gyri are also interconnected by commissural paths through the corpus callosum.

From the region below the left principal fissure, some uncrossed and crossed fibers appear to enter the radiations 
that ultimately separate into the external capsule and extreme capsule. These fibers distribute through external and extreme capsules downward into superior (fig. $4 \mathrm{~A}$ from animal 5 shows this connection), middle, and inferior temporal gyri and hippocampal gyrus. Many of the fascicles extend caudalward in the superior longitudinal fasciculus before turning out into external and extreme capsules. The fibers which have crossed through the corpus callosum from left to right are reinforced by fibers from area 5 entering the external capsule. This occurs after fibers had extended rostralward from the level of the parietal lesion through the superior longitudinal fasciculus, and then turned out from it into the external capsule. These fibers comprise frontotemporal and parietotemporal association and commissural bundles.

Some of the fascicles in the external capsule pass ventral to the lentiform nucleus. This group of cortical fibers distribute successively into the putamen and the globus pallidus as a frontostriate connection (fig. $4 \mathrm{C}$ from animal 5 illustrates this). Many other frontostriate fibers reach the lentiform nucleus through the anterior limb of the internal capsule. A few of this group of corticostriate fibers cross through the corpus callosum before entering the internal capsule. Fascicles that course beneath the corpus callosum from the frontal lobe as constituents of the subcallosal bundle enter the tail of the caudate nucleus at approximately the midpoint of the diencephalon (see fig. 5E from animal 7). As the parietal lesion comes into the field, it contributes to both subcallosal bundles. Both frontocaudate and parietocaudate fibers appear to lose their myelination upon entry into the caudate nucleus (fig. 5E).

As the degenerating fascicles in the external capsule travel ventral to the lentiform nucleus, some fibers approach the anterior commissure and then turn downward into the basolateral portion of the amygdaloid nucleus. Others continue in their ventromedial course to prepyriform cortex and also enter the islands of Calleja, the anterior olfactory nucleus, and the medial septal nucleus. Some corticoseptal fibers from 
the region of the lesion in the frontal lobe swing medialward to interdigitate with the fibers of the corpus callosum and the underlying subcallosal bundle. They follow along the fibers of the precommissural fornix into the medial and lateral septal nuclei. Thus fronto-olfactory pathways establish a dorsoventral association system, similar to those described by others for occipital areas (Bailey et al., '43).

From the region beneath the lentiform nucleus, a small group of degenerating fibers turns around the edge of the internal capsule into dorsal thalamus (shown for animal 5, fig. 4C) and follows along the medial border of nucleus ventralis anterior into stria medullaris thalami as a lateral corticohabenular tract. Also curving around the lower edge of the internal capsule and overlaping the last mentioned bundle are the fibers of the inferior thalamic peduncle (also shown in fig. $4 \mathrm{C}$ from animal 5). These peduncular fibers appear to separate into two divisions as they approach the dorsal thalamus at the dorsomedial border of the internal capsule. The lateral and rostral division enters the nucleus ventralis anterior of the dorsal thalamus. The medial division (illustrated for animal 5, fig. 2A-C), and on the whole the more caudal, passes along the medial border of nucleus ventralis lateralis to the medial thalamic nucleus and to the midline thalamic nuclei. Possibly this division corresponds to the temporothalamic connections described by Whitlock and Nauta ('56) between the inferior and middle temporal gyri and the medial thalamic nucleus, our lesion cutting the bundle in course.

Some corticostriate fibers which reach the globus pallidus by the internal and external capsules terminate in this area. This same connection was seen in animal 5 (fig. 4C). They are accompanied by other fibers of cortical origin which pass through the globus pallidus and the posterior limb of the internal capsule and then converge in the zona incerta. Some terminate in the zona incerta or the nucleus of the field of Forel as a corticoincertal path. A few of these fibers turn ventralward to end in the ventromedial hypothalamic nucleus and the lateral hypothalamic area as frontohypothalamic 
fibers. The remainder appear to descend to midbrain tegmentum as corticotegmental fibers. These last are scant in number.

Degenerating fascicles pass from the parietal area 5 to the superior parietal convolution of the contralateral hemisphere through the corpus callosum. After crossing, many of these fascicles extend rostral to their region of decussation. They are similar to the association and commissural paths found for the frontal cortex. Many fibers pass through the posterior limb of the internal capsule, the cerebral peduncle and the base of the pons along with frontal projections as components of the parietopontine and pyramidal tracts. This last finding confirms the projection of area 5 through the pyramidal system (Peele, '44). From area 5 fibers, also entering the posterior limb of the internal capsule, pass in a diffuse manner through the external medullary lamina into the nucleus ventralis lateralis of the dorsal thalamus. There is indication that a very few fibers terminate in the medial nucleus (Peele, '44). There is definite evidence for the existence of fibers traveling ventrocaudalward along the outer border of the nucleus reticularis, then crossing the medial lemniscus as it enters the dorsal thalamus, and traversing the zona incerta to reach the red nucleus. These are corticorubral fibers from the superior parietal area (area 5). There is an appreciable number of the degenerating fibers from the lesion in the parietal area that enters the postlenticular portion of the internal capsule. A portion of them turn into the pulvinar and the tegmentum of the midbrain. Those entering the pulvinar course through the posterior commissure to enter the medial longitudinal fasciculus. They also appear to end in the oculomotor complex and the caudal part of the red nucleus.

\section{Protocol 3}

Animal 3, a female Macaca mulatta weighing approximately $6 \mathrm{lb}$, was operated upon on $1 / 20 / 56$. The left frontal bone was removed in the region of the supraorbital ridge for an area extending caudalward to the coronal suture, medialward to the midline, and lateralward to the origin of the temporalis 
muscle. Following the incision of the dura, areas 9,46 and 8 above the principal fissure of the left superior frontal convolution were ablated (fig. 5A).

During the two weeks of postoperative observation, the monkey tended to hold the right upper extremity in flexion at shoulder, elbow, wrist, and phalangeal joints. Coordinated, fine voluntary movements were made, however. There were no other noticeable deviations in cardinal signs, level of consciousness, sensory modalities, or motor activities.

Gross examination of the brain at necropsy demonstrated a lesion in the left superior frontal convolution, in area 8 above the principal fissure, and in areas 9 and 46. Marchi technique used with coronal sections of the brain documented the gross extent of the lesion and also showed its continuation into the corona radiata and the cortex rostral to the cingulate gyrus. Degeneration was not apparent rostral to the points of the gross lesion.

From the site of the lesion, degenerating fibers can be followed ipsilateralward and, by way of the genu and rostral end of the corpus callosum, contralateralward to area 45 below the principal fissure and to the frontal pole at the level of the lesion. The degeneration is most evident in ipsilateral area 45. Degenerating fibers continue from the lesion into the cingulum of the ipsilateral side to distribute to the cingulate gyrus above the corpus callosum. Others cross through the genu of the corpus callosum to enter the contralateral cingulum and to be distributed to the cingulate gyrus. However, the greater part of fibers extends through the body of the corpus callosum to areas 8,9 and 46 of the side opposite to the origin of the fascicles. These association and commissural tracts are similar to those described as richly interconnecting the areas $8,12,45$ and 46 in protocol 2. A portion of the fibers form crossed and uncrossed components of the external and extreme capsules, and then distribute ventrocaudalward into the insula, the orbital gyri, the superior, middle, and inferior temporal gyri and the hippocampal gyrus. The fibers constitute intrafrontal and frontotemporal association and commissural pathways. 
As the anterior limb of the internal capsule appears in the series, degenerating fibers from the lesion (probably from area 8) enter this limb to reach the caudate nucleus, the putamen and the globus pallidus, successively, as corticostriate fibers. These are illustrated for animal 7 (fig. 5E). A few enter the body of the corpus callosum to be distributed to the contralateral striatum. Some crossed and more uncrossed fibers either turn into the internal capsule or bypass it to enter the radiations that separate to become external capsule and extreme capsule. Those following the external capsule extend ventralward into the lentiform nucleus to be distributed to it as frontostriate fibers. These are shown for animal 5 in figure $4 \mathrm{C}$.

As the fibers are followed ventralward into the lentiform nucleus, some are observed to end in substantia innominata (fig. 4C, animal 5) and others continue to the septal region. The presence of fine granules along the lateral olfactory tract and the anterior commissure suggest that fascicles extend into the pyriform lobe. A very few granules in the precommissural fornix suggest that small fascicles pass through it into the parolfactory area as corticoseptal tracts.

Many of the degenerated fibers evident in the anterior limb of the internal capsule turn across the thalamus to end in the midline nuclei and the parvocellular portion of the medial nucleus. Some of the fibers enter the dorsal thalamus at the level of the thalamic fasciculus. These can be traced to the nucleus ventralis lateralis of the dorsal thalamus (shown for animal 5 in fig. 2B). Scattered degenerating fibers of the anterior thalamic radiations, after crossing in the anterior limb of the internal capsule, pass to the nucleus ventralis anterior or extend through it or through the lamina of the anterior nuclear group of the dorsal thalamus. Some of these fibers, at least, reach the midline to enter the diencephalic periventricular system, which is illustrated for animal one (fig. 3A). Periventricular fibers can be followed lying close to the third ventricle into paraventricular, dorsomedial, and ventromedial hypothalamic nuclei. These form corticothalamic and small corticohypothalamic tracts. 
A portion of the fibers which have passed through the internal capsule appears to turn downward toward the midbrain through zona incerta to the rostral midbrain periventricular gray and the tegmentum dorsolateral to the red nucleus. They are cortico-incertal and corticotegmental bundles. These corticotegmental fibers can be followed into the red nucleus, forming a corticorubral tract, and into the region of the interstitial nucleus of the medial longitudinal fasciculus, the cortico-interstitial bundle of Muskens ('14).

\section{Protocol 5}

A small male Macaca mulatta, about $8 \mathrm{lb}$ in weight, with a left lower extremity missing below the knee, was operated upon on $2 / 21 / 56$. A portion of the right inferior frontal convolution was ablated. When the animal had awakened, his movements were relatively well coordinated. A counterclockwise circus motion dominated his motor activity for 5 days following the surgery. Coincident with this, there were a diminution in food and water intake and an extensive edema of the face. During three weeks of observation, the motor pattern and eating habits regained their preoperative status and the swelling of the face disappeared. This monkey was more tractable following the surgery.

On gross examination, the ablation of areas 12 and 13 of the right inferior frontal convolution was confirmed (fig. 5B). Study of the Marchi slides revealed extension of the lesion on the right side into approximately the rostral one-half of the caudate nucleus, the rostral one-half of the putamen, the rostral one-fourth of the claustrum, the septum pellucidum, the septal area, the anterior limb of the internal capsule, and the rostrum of the corpus callosum.

From the orbital gyri, degenerating association fibers can be followed as they ascend through the external capsule and the extreme capsule into the white matter to reach all convolutions of the frontal lobe rostral to the level of the lesion. These fibers lose their myelin in the pyramidal layer of the cortex. Some fibers, after leaving the external and extreme 
capsules, swing medialward to cross through the corpus callosum to the contralateral frontal gyri. Some degenerating fibers of this group coursing in the corpus callosum turn downward into the subcallosal gyri and gyri recti by way of the rostrum of the corpus callosum. The insula and the cingulate gyrus also receive degenerating fascicles bilaterally. These areas on the side of the lesion are supplied by fascicles directly out of the upper borders of the external and extreme capsules. The cingulate and insular regions on the side opposite the lesion are supplied after the fibers have crossed in the corpus callosum and, for the insula, swing ventralward through the extreme capsule.

There are also fibers extending from the external and extreme capsules, particularly the latter, to the temporal pole, the superior, middle and inferior temporal gyri and the hippocampal gyrus on the side of the lesion and on the contralateral side. Proportionately more degeneration is evident in the homolateral inferior portion of the superior temporal gyrus (fig. 4A) than in any other temporal region. Thus, numerous association and commissural tracts connect the inferior part of the orbitofrontal area bilaterally to all other parts of this orbitofrontal region, to the cingulate gyrus, to the insula, and to the rostral portions of the temporal lobe, including its pole.

Corticoseptal fibers are observed in the induseum griseum, precommissural fornix and septohippocampal nucleus of both sides. Like the previously described group of fibers, these travel through the external and extreme capsules and then, by way of the white matter of the hemisphere, forward to the midline where they undergo a partial decussation in the corpus callosum. Before, or after crossing, they interdigitate with the fibers of the corpus callosum to reach the region of the precommissural fornix. They accompany fibers of this portion of the fornix into the parolfactory area (medial and lateral septal nuclei), or extend back into the region of the septum pellucidum along the septohippocampal nucleus, or follow around the genu of the corpus callosum into the por- 
tions of induseum griseum ventral to the genu of the corpus callosum.

A small but discrete bundle, with an entirely different course, can be followed from the level of the lesion ventralward through the external capsule to the lower border of the lenticular nucleus. Here some of the fibers extend directly rostralward to the anterior perforated space (tuberculum olfactorium). These corticotubercular (see fig. 6C from brain of animal 7) and corticoseptal fibers can be traced to the islands of Calleja, the diagonal band of Broca and its nucleus, the anterior olfactory nuclei, and, more dorsocaudally, to the medial and lateral septal nuclei or parolfactory area. The medial forebrain bundle can be followed from the medial septal nuclei to the ventromedial hypothalamic nucleus, the lateral hypothalamic area and the infundibular part of the hypothalamus. There is degeneration from the external capsule into the anterior commissure and into the supracommissural components of the right stria terminalis which extend into the bed nucleus of the anterior commissure. Since the lesion has cut across the external and extreme capsules and the medial forebrain bundle and intruded into the septal area, a portion of this degeneration cannot be attributed to the orbitofrontal lesion. However, some of the pattern gives support to the previous evidence for cortico-olfactory connections to the basal parts of the hemisphere through the external capsule.

Accompanying the corticotubercular fibers are small degenerated bundles which turn out from the external capsule into the globus pallidus; others ascend in the external capsule to enter the internal capsule and thence to reach putamen and globus pallidus. By whichever course they travel, these are partly corticostriate fibers and partly corticotegmental fibers. Commissural connections yield corticostriate fibers to the contralateral lentiform nucleus.

From the inferior frontal convolution, fibers which have passed upward through the external and extreme capsules toward the corpus callosum can be observed in both subcallosal 
bundles (fig. 5E shows this for animal 7). These fibers travel some distance caudalward to enter the tail of the caudate nucleus bilaterally. Additional fibers extend from the right caudate nucleus over the corpus callosum to the left caudate nucleus, streaming around the anterior horn of the lateral ventricle to enter the caudate nucleus medially and laterally. Internuclear connections from the right caudate nucleus to the lentiform nucleus (fig. $4 \mathrm{~B}, 4 \mathrm{C}$ ) can be traced as degenerating fascicles that cut across the internal capsule.

Some of the fibers from the caudate nucleus collect laterally to turn across the edge of the anterior thalamic radiations. They reach the medial nucleus of the dorsal thalamus by passing over the external medullary lamina and through either the zonal layer of the dorsal thalamus or the nucleus ventralis anterior to attain, and then pass through, the internal medullary lamina. From the anterior thalamic radiations many fibers appear to pass through the anterior nuclear group and nucleus ventralis anterior of the thalamus to terminate, in large part, in the medial nucleus of the dorsal thalamus (fig. 2, A to C). Supposedly these represent the corticothalamic constituent of the anterior thalamic radiations to the nucleus medialis, but the available material does not establish their cortical origin, since the lesion is in the anterior limb of the internal capsule. Fibers from the lentiform nucleus pass across the internal capsule along the thalamic fasciculus and into nucleus ventralis lateralis and centrum medianum homolaterally. There are fibers in the inferior thalamic peduncle (fig. 2B. See also fig. 4C) which run upward along the edge of the internal medullary lamina to the medial nucleus of the thalamus.

Many of the fibers enter the globus pallidus (fig. 4C), converging at its medial border to cross the posterior limb of the internal capsule and reach the zona incerta and the nucleus of the field of Forel. Some of these fascicles turn caudalward to enter the tegmentum of the midbrain where they can be traced into the region of the substantia nigra, the medial longitudinal fasciculus, the oculomotor nuclei, and the dorsal part of the deep tegmental gray. 
A great many homolateral and a few contralateral fibers that have traversed the internal capsule are present in the cerebral peduncles in the position of the frontopontine tracts. Some of these frontopontine fibers appear to continue farther caudalward in the superficial stratum of the pons.

\section{Protocol 6}

A male Macaca mulatta, weighing about $6 \mathrm{lb}$, was operated upon 4 times. Cortical ablations were carried out (fig. 6A) and the medial nucleus was stimulated as described below.

On $3 / 23 / 56$ left area 6 (fig. $5 \mathrm{~A}, 5 \mathrm{C}$ ) the premotor frontal cortex was destroyed rostrally along with the anterior twothirds of the portion of area 8 adjacent to the superior ramus of the arcuate fissure. In the subsequent two months of observation a number of the changes noted postoperatively persisted. These included a bilaterally uniform pilo-erection (fig. 1G) from the level of the 12th rib upward, increased food and water intake, increased sweating, sialorrhea, and accentuated purposeless motor activity. Sudoriferous secretion was tested by cleansing hand pads, foot pads, and perinasal and perioral skin with alcohol. The surfaces were allowed to air dry. Quinizerine powder was applied. This gray powder turns markedly purple in the presence of moisture. Within three minutes all of the cleansed areas showed evidence of perspiration. Random comparisons were made with 4 other animals, preoperatively, postoperatively and in the interim of observation. It was usual for some suggestion of moisture to appear on the hand and foot pads by the end of 5 minutes. This animal maintained a comparatively higher level of sudoriferous activity throughout two months. For at least three weeks, it was possible to demonstrate catalepsy (fig. 1G) in this monkey as he was handled. He was reluctant to use the right upper extremity for a week following the experiment. During this same period there was a generalized tremor present.

On $4 / 16 / 56$ the left medial nucleus of the thalamus was stimulated. A higher strength of current than previously 
employed was required to produce clonic movements of the contralateral extremities. The movements obtained were diminished in strength and amplitude in comparison to previous experimental results. Cortical ablation of the left areas 4, 3 and 2 (fig. 5A) controlling upper and lower extremities was performed. Following this procedure the animal had increased difficulty in manipulation of the right upper and lower extremities. Although this condition improved, the awkwardness persisted for two months. However, the difficulty in maintenance of balance did disappear in one week. This monkey was irritable after this portion of the experiment.

On $4 / 27 / 56$ the left medial nucleus of the thalamus was stimulated. The results of stimulation observed previously were seen again in this instance. The monkey became extremely cold to touch during the stimulation. Body temperature was elevated within 40 minutes following the cessation of the surgical procedure. Respiration was slow and stertorous during the nuclear stimulation. When the inferior tip of the left area 1 and the superior border of area 22 in parietal and temporal lobes, respectively, were destroyed (figs. 5A and $6 \mathrm{~A})$, respiration depressed its excursions. There was a return to the preoperative status as body temperature improved. The irritability present during the second portion of this experiment disappeared at this point. Right upper and right lower extremities were held stiffly in flexion at shoulder and hip, elbow and knee, wrist and ankle, metacarpal-phalangeal and metatarsal-phalangeal articulations and interphalangeal articulations.

On $5 / 11 / 56$ the left medial nucleus of the thalamus was stimulated. The results of stimulation recorded previously were repeated at this time, also. Again there was a change of respiration to a slow and stertorous type during stimulation. Concomitantly, the animal felt cold to touch and shivered with the nuclear stimulation. Body heat was regained within 30 minutes following the operative procedure. Cortical ablation of the left area 6 (figs. 5A, 5C, $6 \mathrm{~A}$ ) below the inferior ramus of the arcuate fissure and the orbital gyri on the left frontal 
lobe of the cerebral cortex was performed. Postoperatively, there was some decrease in the tonicity of the right upper and right lower extremities and a diminished patellar reflex on the right side. Otherwise, there were no noticeable differences from the preoperative state.

The degeneration from areas 6 and 8 can be followed into the genu and body of the corpus callosum. It extends into both internal capsules and into the complex that will become external and extreme capsules. This complex is supplemented by a substantial number of fibers from the sites of the lesions in lower area 6 and the orbital gyri. The dual mass of degenerating fibers extends as a part of the diagonal band of Broca into the anterior perforated space (see fig. $6 \mathrm{~B}$ and $6 \mathrm{C}$ for animal 7) and the parolfactory area of both sides. In these regions there is bilateral degeneration in the medial septal nucleus, the diagonal band of Broca, the islands of Calleja, and the anterior olfactory nucleus. There are some fibers that enter nucleus accumbens (see also fig. $6 \mathrm{~B}$ and $6 \mathrm{C}$ for animal 7 ) and putamen, bilaterally. On each side this degeneration extends lateral and caudal to the substantia innominata. A few fascicles appear to pass through the corpus callosum and follow the precommissural fornix into the lateral septal nuclei. Possibly they are septohippocampal in course and are part of the perforating bundles to the region of induseum griseum.

As the extreme and external capsules begin to form, the degeneration is apparent in the homolateral claustrum and insula. Degenerating fascicles from these capsules can be found in the superior, the middle, and the inferior temporal gyri on both sides. There is degeneration in small amount to the homolateral corticomedial and basolateral amygdaloid nuclei and, in larger amount, to the comparable nuclei of the other side by way of the external capsule and then the anterior commissure.

Some cortical fibers enter the globus pallidus from its ventral aspect; they are degenerated also in the brain of animal 5 (fig. $4 \mathrm{C}$ ); others appear to continue directly into the ansa lenticularis. Some of the latter can be traced into the lateral 
hypothalamic area, and are lost in the medial hypothalamic region. Many of the fibers accompanying the ansa join the ventral capsule of the subthalamic nucleus and appear to enter this nucleus. There is marked degeneration in the ventrolateral and medial nuclei of the dorsal thalamus, indicating that the cortical areas ablated give rise to corticothalamic fibers. It should be pointed out that at the present time the observer is certain, on the basis of more limited lesions, that some of these corticothalamic fibers arise in areas 6 and 8 and the orbital region, but cannot exclude the possibility that some of them have origin from the inferior frontal gyrus or from portions of areas 4, 3 and 2. Some of the fibers which entered the globus pallidus traverse it to enter the lenticular fasciculus and accompany it across the posterior limb of the internal capsule and medialward through the zona incerta, where some of them end in the nucleus of the field of Forel. A very few granules can be seen along the more caudal course of the lenticular fasciculus in the tegmentum of the midbrain and in the nucleus of the medial longitudinal fasciculus. A few granules can be traced through the posterior commissure into the medial longitudinal fasciculus and the oculomotor nucleus of the other side. There are degeneration granules in the homolateral medial longitudinal fasciculus and the oculomotor nucleus. They are very few in number, but the constancy of their position from section to section suggests they are not artefacts.

From the ablated areas in the frontal lobe rostral to area 4, degenerating fibers have collected in the anterior thalamic radiations. They enter through the upper edge of the reticular nucleus and external medullary lamina to end in the nucleus ventralis lateralis. They are also seen in the brain from animal 5 (fig. 2B). The fibers reaching the nucleus ventralis lateralis are especially prominent when the lesion in area 4 begins to contribute fibers to the internal capsule of both sides. A very few of the fibers, either by crossing the nucleus ventralis anterior or by traversing the zonal layer, collect in the internal medullary lamina and then enter the dience- 
phalic periventricular system; they appear to end in the midline thalamic nuclei and the paraventricular hypothalamic nucleus. A large proportion of the degenerating fibers in the internal capsule can be traced to the left corticospinal projection in the cerebral peduncle and the base of the pons.

\section{Protocol 7}

A female Macaca mulatta, weighing approximately $6 \mathrm{lbs}$, was operated upon 5/20/56. A this time, the left medial nucleus of the thalamus was stimulated with resulting clonic movements in the contralateral portion of the body according to the pattern which has been described earlier.

On 5/1/56 stereotaxic stimulation and ablation of the right globus pallidus (fig. 5D), and then, on 5/18/56, of the left globus pallidus, was carried out. Hypertonicity with cogwheel rigidity was present in the movements obtained with caudal stimulation points. Face and arm movements seemed to dominate at the rostral stimulation points; leg movements were present, although not dominant, in the caudal stimulation points. Athetoid positions and tremor were elicited at the midpoint in the stimulation. Most of these movements were flexion and internal rotation involving the articulations of the upper and lower extremities on the contralateral side. There was a drop of body temperature at the midpoint of stimulation. Immediately postoperatively, the animal tended to hold the homolateral extremities in flexion and the contralateral extremities in extension. All extremities were hypertonic. There was a tendency for the head, the neck, and the eyes to deviate toward the side of the lesion. Following both operations the animal felt cold to touch and shivered for about 30 minutes. A check of body temperature after the second procedure showed a rectal temperature of $36.2^{\circ} \mathrm{C}$. This was about 11:00 A.M., at an hour when the monkey will generally have a rectal temperature of $38.3^{\circ} \mathrm{C}$. Oral movements such as a homolateral smacking of the lips and protrusion of the tongue to the side of the stimulation were present. During the month of further observation there were no outstanding 
features other than sustained lethargy and fear. It was extremely difficult for the examiner to entice the animal out of a crouched position in the corner of the cage and, when movements were finally made toward food, they were performed slowly.

At necropsy there were healed superficial hemorrhagic lesions of the left area 6 and the right area 4 of the frontal lobe. Marchi slides indicate that the stereotaxic lesions include the internal capsule, the tail of the caudate nucleus, the globus pallidus, the nucleus ventralis anterior, and the nucleus ventralis lateralis of the dorsal thalamus on both right and left sides. The left medial nucleus shows evidences of electrode paths. The external and internal divisions of the left globus pallidus contain discrete electrode lesions (fig. 5D). Roughly one-fourth of the caudal portion of the basal ganglia is involved in the lesion on each side.

Study of the Marchi slides shows degenerating fibers from the superficial hemorrhagic areas. These fibers are components of association tracts which enter the adjacent homolateral gyri of the frontal cortex and pass to the contralateral frontal gyri after having decussated through the genu or body of the corpus callosum. There are conspicious bundles of fibers passing to the cingulate gyri from both of these lesions. This material, correlated with that from protocol 3, suggests that the projection from area 6 , at least, is bilateral to the cingulate gyri.

Degenerating fascicles from the cortical lesions enter both subcallosal bundles and thence pass into the head of the caudate nuclei (fig. 5E). These are certainly from area 6 (see Protocol 3) and possibly from area 4. A considerable number of degenerating fibers follows through the corona radiata into external and extreme capsules to reach the orbitofrontal area. Again, bundles can be traced into the putamen, the nucleus accumbens (fig. $4 \mathrm{~A}$ ) the anterior olfactory nucleus, the islands of Calleja, and the diagonal band of Broca (fig. $6 \mathrm{~B}, 6 \mathrm{C})$. There appear to be fibers from the corpus callosum interdigitating with the precommissural fornix to terminate 
in the lateral and medial septal nuclei (parolfactory area) or to continue into the right and left preoptic areas. These are stria terminalis fibers in part. As in the preceding experiments, the degenerating fascicles from the external capsule enter the anterior commissure; degenerated anterior commissural fibers which, as has been pointed out, reached this commissure by way of each external capsule, terminate bilaterally in the uncus, the hippocampus, the amygdaloid complex, and the inferior temporal gyrus. Degeneration granules ean be followed into the induseum griseum from the anterior continuation of the hippocampus and septohippocampal nuclei. The induseum griseum on the left is more affected.

Rather heavily myelinated fibers arising from the area of the lesion in each globus pallidus constitute the pallidohypothalamic tract (Ranson, Ranson, and Ranson, '41), which terminates primarily in the ventromedial hypothalamic nucleus (fig. 3B). Along their course, they appear to send some fibers to the medial and lateral hypothalamic areas.

There is a large amount of degeneration in each globus pallidus. The degenerated fascicles form components of the inferior thalamic peduncle. A portion of these fibers follows the ansa lenticularis toward the ventricle but turns upward into the dorsal thalamus to join fascicles from the thalamic fasciculus. Their termination seems to be principally in the nucleus ventralis lateralis and the medial nucleus of the dorsal thalamus. Degenerating strands of fibers, believed to be from the caudate nucleus, can be seen in the anterior thalamic radiations and followed across the external medullary lamina and through the stratum zonale of the dorsal thalamus to the internal medullary lamina. These end in course in the nucleus reticularis, the nucleus ventralis lateralis, and the medial nucleus of the dorsal thalamus. Possibly they reach the anterior nuclear group (Sachs, '09).

The majority of the fibers from the globus pallidus which have collected in the ansa lenticularis and fasciculus lenticularis turn downward through the field of Forel where many 
of them end. Others extend into the tegmentum of the midbrain (the red nuclei and the oculomotor nuclei), or enter the posterior commissure or the medial longitudinal fasciculus.

Pyramidal and corticopontine fibers passing in the internal capsule can be traced into the cerebral peduncles and the base of the pons. Their degeneration was due to an involvement of the posterior limb of the internal capsule in the lesion.

\section{Protocols 9 and 11}

These animals will be considered together since they represent a similar phase of the experimentation. Animal 9, a male Macaca mulatta weighing approximately $6 \mathrm{lb}$, was operated upon on $7 / 13 / 56$. A large semicircular skin incision was made, the subcutaneous tissue reflected, and the temporalis muscle sectioned on the left side. Bone was removed from the supraorbital margin to the sagittal suture and from the superior nuchal line to the zygomatic process in order to expose the left hemisphere of the brain. Cerebral cortex was destroyed by manual dissection and electrocautery leaving behind the corpus striatum and the corpus callosum. The cavity was loosely packed with gelfoam, the dura mater repaired, the temporalis muscle sutured to subcutaneous tissue, and the skin stitched and covered with a sterile dressing. Seven hundred and fifty cubic centimeters of Ringer's solution as intravenous infusion were used during the procedure.

Animal 11, a male Macaca mulatta weighing $16 \mathrm{lb}$, was operated upon on $11 / 6 / 56$. The cerebral cortex was removed from the right hemisphere down to the corpus striatum. The repair and intravenous infusion were comparable to the procedure described for animal 9. Although respirations were paradoxical for about 6 hours following the surgery, the postoperative recovery was not eventful.

Both animals refused food and water for approximately two days postoperatively. When they began to eat, their intake was, and remained, above the normal level for other animals observed. In each there was a large amount of periorbital and facial edema, which subsided within 4 days after the 
surgery. Although the animals were able to right themselves by the third day, the side contralateral to the hemispherectomy was paretic and flaccid. As the animals began to move, a circling motion toward the side of the lesion appeared and is sustained to this date. It is a dominant part of the motor activity of these monkeys. It can be demonstrated that a contralateral homonymous hemiopsia exists in each monkey. Extensor muscular tone began to return to the paretic extremities after 7 days in animal 9 and after 13 days in animal 11. When sudden movemets occurred in the handling of either animal, a spasmodic and clonic extension was initiated in the extremities, the head, and the neck. Both animals began to use extremities on the paretic side within 20 days, postoperatively. The movements involved essentially the whole arm and the whole leg.

Stimulation of the left and the right medial thalamic nuclei was carried out in animal 9 one month following the hemispherectomy (fig. 1, D-1 and D-2). This was done under operative aseptic technique in the manner described in the first protocol. A concentric electrode was used. Double the strength of stimulation up to $25 \mathrm{v}$ was required to elicit response from the left nucleus in comparison to a $10 \mathrm{v}$ to $14 \mathrm{v}$ stimulus strength employed for the right medial thalamic nucleus. The pattern of movement that has been recounted for the thalamic nuclear response to stimulation (p. 265) was present. Following surgery, the monkey's body temperature dropped $1.2^{\circ} \mathrm{C}$ and the animal shivered for 20 minutes. There was a marked increase in salivation.

Similar stimulation was performed on animal 11, 4 months after the hemispherectomy. A monopolar electrode used to stimulate the right medial thalamic nucleus brought out the pattern of movements which has characterized this nucleus (fig. 1, A-D). Following surgery there was a marked sialorrhea and a fall in body temperature.

Two weeks following this stimulation, on $2 / 26 / 57$, the basal ganglia were removed on the decorticated side. On $3 / 19 / 57$, when the medial thalamic nucleus was stimulated, no move- 
ment could be produced on the contralateral side. It was apparent that the tonicity of the musculature was increased, but the body parts remained inactive. By directing the electrode across the midline, the left medial thalamic nucleus was stimulated with a repetition in the pattern and strength of stimulus usually required. At necropsy the decorticated hemisphere showed the putamen, the globus pallidus and a portion of the candate nucleus to be lacking.

\section{CORRELATION AND DISCUSSION OF ANATOMICAL AND EXPERIMENTAL RESULTS}

The observable results accruing from the stimulation of the medial nucleus of the thalamus represented combined somatic and visceral motor activity with an emphasis on the former. Destruction of the nucleus shifted the emphasis to autonomic signs, although both varieties of behavior were present. In order to interpret these results, the afferent and efferent neuronal connections of the medial nucleus of the thalamus will be considered. It seems desirable to organize this multiplicity of detail into ascending connections, internuclear connections, interdiencephalic connections, striatal connections, and, lastly, cortical connections. The approach made to this experimental problem does not contribute any information to that already present in the literature with regard to the ascending connections. Therefore, they are not considered in this discussion.

\section{Internuclear connections}

It was possible to follow degeneration from the small stereotaxic lesions in the medial nucleus across the internal medullary lamina into the nucleus ventralis lateralis (fig. 3A) and the nucleus ventralis posterior. Our material was not suitable for determining those afferent to the medial nucleus. However, Crouch and Thompson ('38) and Crouch ('40) concluded from their material that all parts of the lateral and ventral nuclei were interrelated to the medial nucleus. Other observers who have noted these connections are Sachs ('09), 
Rioch ('29), Le Gros Clarke ('32), Walker ('38), and Mettler ('47a, '47b). Such a relationship allows the ascending somatic and visceral sensory impulses to be available to the medial nucleus.

Degeneration of intrathalamic fibers from the medial nucleus to the anterior nuclear group was believed to be present, but not demonstrated unequivocally because it was impossible to dissociate these fibers from the electrode lesion which passed through them (protocol 1). However, fibers could be followed from the medial nucleus along the anterior ramus of the internal medullary lamina into nucleus anteroventralis (protocol 1). Ariëns Kappers, Huber and Crosby ('36) and Rioch ('29) described rich connections from the medial nucleus to nucleus anteroventralis.

Fascicles from the medial thalamic nucleus to the midline nuclei were traced by way of the diencephalic periventricular system (protocol 1; fig. 3A). They have been found previously for primates as indicated by Ariëns Kappers, Huber and Crosby ('36) and Crosby and Woodburne ('51).

The degenerating intrathalamic fibers which traversed the internal medullary lamina from the medial thalamic nucleus to ventral and lateral thalamic nuclei declined in quantity as they emerged from the lamina (fig. $3 \mathrm{~A}$ ). On this basis some assumption can be made that the fascicles terminate in the nucleus paracentralis, the nucleus centralis lateralis, and the centrum medianum. These internuclear fibers to intralaminar nuclei are similar to those mentioned by Huber and Crosby ('29) and Sheps ('45). Considered collectively, the internuclear pathways reinforce the inflow of somatovisceral afferent impulses carried by the ascending sensory systems.

\section{Interdiencephalic connections}

In this study the Marchi granules along the lightly medullated fibers of the ablated medial thalamic nucleus extend rostromedially to its oral tip, then into the diencephalic periventricular system (fig. 3A). There is successive diminution in the degeneration as the fibers course downward through 
the midline thalamic nuclei, the periventricular hypothalamic nucleus and the dorsomedial and ventromedial hypothalamic nuclei (protocol 1). This well organized series of connections is deseribed in the papers by Le Gros Clarke ('32), Ariëns Kappers, Huber and Crosby ('36), and Ingram ('40) and, in a fashion particularly pertinent to this problem, in that by Crosby and Woodburne ('51).

The nuclei of the habenulopeduncular tract are in synaptic relationship with the medial thalamic nucleus (Ariëns Kappers, Huber and Crosby, '36). Such an arrangement will permit an additive influence of olfactory impulses from corticoolfactory connections (protocols $2,3,5,6,7$ ) that secondarily reach habenula through the stria medullaris thalami.

By means of these interdiencephalic connections, the medial nucleus is in a position to influence the autonomic nervous system outflow from the hypothalamus over both the craniosacral and thoracolumbar divisions of the system. Thus it can modify visceral behavior by somatic influences. Conversely, the hypothalamus contributes a visceral component to the medial thalamic nucleus.

It seems appropriate to recall certain other features indicated in the functional connections of the hypothalamus, since variations in the autonomic responses appear in our experimental series. The anterior hypothalamic area regulates against heat (Ranson, '40) and possibly the posterior hypothalamic area regulates against cold (Ranson, '40). The mammillary peduncle is thought to bring visceral sensibility forward to the medial hypothalamic area from the gray of the fasciculus solitarius (Allen, '23). Olfactory impulses relayed over the fornix system and the medial forebrain bundle distribute throughout preoptic and medial hypothalamic regions with a major discharge of the fornix to the mammillary body. Recent evidence by Scharrer ('54), Mirsky, Stein, and Paulish ('54), Porter ('54), and Roberts and Keller ('56) indicates that the supraoptic and paraventricular nuclei contain neurosecretory cells related functionally to the posterior pituitary lobe hormones. In addition, the posterior pituitary hormones 
may cause the release of ACTH (adrenocorticotropic hormone) from the adenohypophysis during stress. The mammillothalamic tract and the diencephalic periventricular system relay olfactovisceral impulses back to the dorsal thalamus, principally to the medial nucleus. In these many ways the medial nucleus is in a strategic position to receive sensory information from the hypothalamus and to regulate hypothalamic activity. The hypothalamus is generally recognized as affecting glands of internal secretion, and as concerned in the regulation of vasomotor and temperature mechanisms and of salivation and peristalsis. It is an important region in maintaining normal fluid balance and is related to overt manifestations of emotion.

\section{Striatal connections}

The intrastriate connections between the caudate, the putamen and the globus pallidus were abundant (protocol 5). Many such fibers passed from caudate nucleus to the putamen and toward the globus pallidus across the internal capsule. In addition, a group of fascicles connects the head of the caudate nucleus of the one side to that of the other side, passing through the corpus callosum (protocol 5).

The magnocellular portion of the medial nucleus of the thalamus sends fibers to the caudate nucleus (protocol 1 ). The fibers of the medial thalamic nucleus aggregate ventrally in the nucleus to enter the inferior thalamic peduncle on their way to the lentiform nucleus (protocol 1). Similar thalamostriate paths have been found by Papez ('38a, '38b), and Powell and Cowen ('56).

Fibers from the caudate nucleus were traced to the magnocellular portion of the medial thalamic nucleus (protocols 5, 7 and fig. 2, A-C). There are other striatothalamic connections from the ventral surface of the lentiform nucleus where fascicles enter the inferior thalamic peduncle to pass to the medial thalamic nucleus and to centrum medianum (protocols 5,7 ). From the dorsomedial surface of the lentiform nucleus fibers follow the thalamic fasciculus into the nucleus ventralis 
anterior and the nucleus ventralis lateralis. Descriptions of such striatothalamic pathways have been given by Sachs ('09), Huber and Crosby ('29), Ariëns Kappers, Huber and Crosby ('36), Woodburne, Crosby and McCotter ('46), Laursen ('55), and Powell and Cowan ('56).

The discharge pathways from the striatum include ansa lenticularis, fasciculus lenticularis, fasciculus subthalamicus and striatonigral tracts. These discharge paths allow the extrapyramidal motor systems from the cerebral cortex to transmit their impulses downward to motor centers of the brain stem and the spinal cord. The connections to the brain stem are demonstrated for relaying impulses through the gray associated with the medial longitudinal fasciculus (interstitial nucleus) and those to the spinal cord by striatal connections to the tegmental region of the midbrain giving origin to rubrospinal, rubrobulbar, and tegmentospinal tracts (protocols 3 , $5,6,7)$.

\section{Cortical connections}

It is possible to interpret the experimental results in a fashion which permits the grouping of the connections. Those which interrelated parts of the telencephalon include intercortical association and commissural paths, corticostriatal paths and cortico-olfactory paths. The others which will be presented are corticothalamic, corticohypothalamic, corticotegmental, and thalamocortical tracts.

An extensive network of intrafrontal association and commissural tracts relate the superior, middle and inferior frontal convolutions along a vertical axis (protocols 2, 3, 5, 6, 7). From the same regions of the cortex fibers pass by way of the corpus callosum to the comparable frontal area of the opposite hemisphere (protocols $2,3,5,6,7$ ). The regions below the principal fissure send their association and commissural paths upward by way of the external and extreme capsules (protocols 2, 3, 5, 6, 7). The regions above the principal fissure transmit fibers downward through the external and extreme capsules to the ventral and lateral regions of the frontal cortex. Among the regions examined, the medial and 
inferior frontal cortices had the richest intrafrontal associations (protocol 5). From these last areas, pathways travel forward in a horizontal axis, as well as upward in a vertical axis, to reach other portions of the orbitofrontal cortex.

Frontotemporal and parietotemporal tracts are likewise plentiful. From all cortical areas investigated (protocols 2, 3, $5,6,7)$, the association paths are evident in the external and the extreme capsules from the orbitofrontal region and the parietal area 5 to the superior, the middle, and the inferior temporal gyri and to the hippocampal gyrus. Such fibers to the medial portion of the temporal lobe traverse the external capsule principally, but those to the lateral portion of the temporal lobe go chiefly by way of the extreme capsule (protocols $2,3,5,6,7$ ).

Frontocingulate tracts were identified as arising from the orbital gyri (protocols 5, 7) and from the region above the principal fissure (protocol 2,6). These carried both association and commissural fibers. Fronto-insular association and commissural tracts are evident in precisely similar relations (protocols 2, 3, 5, 6).

Crossed and uncrossed corticostriatal tracts to the caudate putamen and globus pallidus were traced from orbitofrontal cortex by way of the anterior limb of the internal capsule (protocols 2, 3, 5, 6, 7). The fibers from below the region of the principal fissure ascended in the external and extreme capsules to enter the anterior limb of the internal capsule. Crossed and uncrossed corticocaudate tracts, from orbitofrontal cortex and parietal area 5 , reach the caudate nucleus by way of the subcallosal bundles (protocols 2, 5, 7). Many crossed and uncrossed corticolenticular fibers traverse the external capsule to enter the putamen and the globus pallidus (protocols 2, 3, 5, 6, 7).

Cortico-olfactory fascicles from the orbitofrontal area, traveling by way of the external capsule, distribute, partly after decussation, to the pyriform cortex, the tuberculum olfactorium, the septum ventromedial to the lentiform nucleus, and the ventrally situated amygdala and pyriform lobe (pro- 
tocols $2,3,5,6,7)$. Other small cortico-olfactory bundles reach the parolfactory area and the induseum griseum by interdigitating with the fibers of the corpus callosum (protocols $2,3,5,6,7)$.

Corticothalamic and corticohypothalamic tracts are found along the anterior thalamic radiations and the inferior thalamic peduncle (protocols $2,3,5,6,7$ ). The connections to the magnocellular portion of the medial nucleus are more evident in the anterior thalamic radiations; those to the parvocellular part of the nucleus are greater in number in the inferior thalamic peduncle (protocols 5,7 ). Corticothalamic connections were plotted by Mettler ('47a, '47b) from areas 11, 9, 8, 6 and $4 \mathrm{~S}$ to the medial nucleus. When Niemer and Jimenez-Castellanos ('50) used the physiologic neuronographic method of Dusser de Barenne and MeCulloch they found corticothalamic connections to the medial nucleus from cingulate gyrus and orbitofrontal areas. Their experimental results are documented in part by our material.

Corticotegmental paths from the frontal and parietal areas examined collect medial to the lentiform nucleus to follow along the lenticular fasciculus (with a partial synapse in the nucleus of the field of Forel) into the tegmentum of the midbrain. Small groups of corticotegmental fascicles enter the globus pallidus by way of the anterior limb of the internal capsule. Other small groups reached globus pallidus by way of the external capsule. Both sets of fibers come together in the medial segment of globus pallidus, then cross through the posterior limb of the capsule to enter zona incerta, where they cross toward the ventricle (with partial synapse in the zona incerta) and turn caudalward into the tegmentum of the midbrain (protocols $2,3,5,6,7$ ). Cortical connections then allow an elaborate interrelationship of all incoming sensory information from the thalamus and the hypothalamus, adding to it the olfactory components.

In this study thalamocortical projections could be followed to orbitofrontal gyri over anterior thalamic radiations and through the inferior thalamic peduncle. Equivocal degenera- 
tion was present in the other portions of the frontal and temporal lobes. Walker ('38) suggested that the bundles forward are very finely myelinated and difficult to follow by Marchi technique. Walker ('38), Freeman and Watts ('47), Pribram, Chow and Semmes ('53) described a lamellar projection of the parvocellular part of the nucleus into the orbitofrontal lobe. Pribram and Bagshaw ('53) added a projection from the magnocellular part of the medial nucleus to the anterior temporal region. Mettler ('47b) found comparable thalamocortical paths to the frontal areas 8, 9, 10, 11 and 12. Papez ('38a) described a medial nuclear discharge to the gyrus rectus or area 14 of the frontal lobe. Physiological experiments of Starzl and Whitlock ('52) demonstrated recruitment of electrical activity of the cortex from the diffuse thalamic projection system in which they included the medial nucleus. The regions aroused were the Walker-Brodmann areas 10,11, $12,14,8 \mathrm{~A}, 8 \mathrm{~B}, 9,45,46$ of the frontal cortex (Walker, ' 40 ). There were small parietal area responses from areas 39,40 , 7 and 5, from occipital and preoccipital areas 18 and 19, and from the tip of the temporal pole. Jasper's 1949 study confirmed these relations.

The results of stimulation of the medial nucleus of the dorsal thalamus as recounted in protocol 1 present a pattern of gross somatic movements and certain visceral phenomena that are the product of ascending internuclear and interdiencephalic connections, striatal and cortical connections. The anatomic pathaways for the spread of a general thalamic discharge have been shown. The effect of overdischarge was demonstrated experimentally. When the medial nucleus of the thalamus was stimulated, a generalized convulsion followed strengths above $10 \mathrm{v}$. Thiele ('05) and Sachs ('09) were able to produce somatic movements by stimulation of the medial nucleus. Hunter and Jasper ('49) obtained turning of the head and the body and epileptiform attacks in cats from implanted electrodes in the intralaminar and medial nuclear region of the thalamus. 
The somatic movements are expressed in the neuron arcs constituting the extrapyramidal systems. Such systems arise from motor and second motor areas of the cerebral cortex. The nature of additional motor areas and their discharge paths is concisely detailed by Crosby ('56). Concepts involved in the behavior of the supplementary motor areas are presented by Fulton, Jacobsen and Kennard ('32), Peterson and Bickers ('52), Schneider and Crosby ('54), Travis ('55a, '55b), and Fleming and Crosby ('56). It is characteristic that movements produced by cortical stimulation of these regions tend to be gross, that the response will be in terms of whole parts of the body - the hand, the foot, the head and the neck rather than as fine, small muscular movements of fingers or toes. Stimulations of areas 6,8 , and 5 were done to determine the type of supplementary motor responses (protocol 2). These responses were similar to the general type of movement aroused by stimulation of the medial nucleus of the thalamus.

However, motor responses resulting from the stimulation of the medial nucleus were possible in the absence of homolateral supplementary motor areas. In the animals in which successive cortical ablations of second motor areas were done, the pattern resulting from stimulation of the medial thalamic nucleus remained the same (protocols $2,3,5,6,7$ ). When there was a complete decortication of one hemisphere, this pattern could still be obtained on stimulation of the medial nucleus of the thalamus (protocols 9, 11). The nature of the response from hemidecorticated animals and from those with successive second motor area ablations suggests that there is a reinforcement of the motor activity over the striatal arcs by that normally occurring in the cerebral cortex. Furthermore, smoothness and organization of movement can be attributed to the functioning second motor areas and/or to intact intercortical connections. Increasing strengths of current were required to obtain the medial nuclear response as more supplementary motor areas were removed. In the hemidecorticated animal there was an especially marked tendency for an entire ex- 
tremity to move rather than the individual parts of that extremity. It is apparent that the connections of the nucleus allow a reverberation of any impulse reaching it. Thus it may be possible that the specificity of response within the limits described will decline as the more cephalic portions of the connections are removed. Finally, the response is absent when the continuity of pathways from the medial nucleus through the basal ganglia into extrapyramidal connections is interrupted.

During stimulation of the middle portion of the medial thalamic nucleus, respiratory changes were elicited. First an increased rate, followed by a diminution in rate and augmentation of depth, and finally apnea resulted. Furstenberg and Crosby ('48) have pointed out that the hypothalamic influence on respiration may be indicated by an increased rate from anterior and dorsal hypothalamic areas. The discharge is by way of the dorsal longitudinal fasciculus and hypothalamotegmental tracts to pontine and medullary respiratory centers. Impulses along pathways from the posterior hypothalamic area and the periventricular gray to the brain stem respiratory centers tend to inhibit respiration. Turner ('54) found in his stereotaxic study of lightly anesthetized monkeys that the stimulation of the medial nucleus of the thalamus always produced an apnea.

With stimulation at the midpoint of the medial thalamic* nucleus, increased peristalsis could be felt through the abdominal wall. There was a sialorrhea, a protraction and retraction of the tongue, and facial movements during the stimulation. This could be explained on the basis of thalamohypothalamic connections and their discharge through the dorsal longitudinal fasciculus to the parasympathetic and motor nuclei of the brain stem (Crosby and Woodburne, '51). A marked drop of body temperature, with a subsequent rise within half an hour, could be demonstrated with stimulation and destruction of the medial nucleus. Normal regulation against both heat and cold by hypothalamic centers (Ranson, '40) was altered in our experiments. The animals in which 
medial nuclear destruction occurred evidenced an increase or decrease, rather than a normal food and water intake. A cessation of normal discharge to hypothalamic centers because of morbid cells, or an increase over normal discharge to certain hypothalamic centers because of moribund cells, might elicit such extremes (p. 290).

The possibilities of reinforcement of autonomic responses by reverberating circuits is likewise present. Turner ('54) and Anand and Dua ('56) have shown changes in blood pressure, heart action, and respiration from implanted electrode studies in cats. Corticohypothalamic paths were observed in the present study from orbitofrontal areas (protocols 2, 3, $5,6)$. The overt manifestations of visceral function and emotional reactions have been mentioned in the protocols.

Attention is drawn to the blunting of emotional activity which was present following the destruction of the medial thalamic nucleus. Clinical use of this fact has been made in psychosurgery (Freeman and Williams, '51; Monnier and Fischer, '50; Spiegel and Wycis, '52). In the objective symptom complex, after medial nuclear destruction, there were a number of similarities to certain mental illnesses in which depression is a feature. These include involutional melancholia, shizophrenia, manic depressive psychoses, temporal lobe epilepsy, petit mal epilepsy and persistent psychosomatic conditions. The experimental work has given some anatomical foundation for certain of these symptoms. In the writer's knowledge, one physician has obtained encouraging results in patients with psychosomatic complaints and mild depressive conditions by using anticonvulsant drugs. ${ }^{3}$

\section{SUMMARY}

1. The medial nucleus of the dorsal thalamus of 12 Macaca mulatta monkeys was stimulated by stereotaxic means in 20 individual experiments for a total of 149 times. A consistent pattern of movement was produced in which stimulation of the rostral parts of the nucleus gave rise to lower extremity

\footnotetext{
'Personal communication from Kent E. Martin, M.D., Cincinnati, Ohio.
} 
movements, the middle parts to trunk and respiratory movements, and the caudal parts to upper extremity, head, and neck movements. Stimulation of medial portions of the nucleus gave upper extremity, trunk, head and neck movements and lateral portions to lower extremity responses. These responses were evoked after hemidecortication on the same side of the brain. This pattern of movements was abolished along' with destruction of the homolateral basal ganglia.

2. Associated phenomena of visceral and emotional behavior resulting from the lesions of the medial thalamic nucleus are described and discussed. The effects of overdischarge and underdischarge of the nucleus are correlated with conditions seen in the practice of medicine.

3. An analysis of the anatomical pathways involved was carried out in the material stained by the Marchi technique. Those connections which mediated the experimental results are emphasized in the discussion. Unusual features of the cortical connections are emphasized. The components of the external and extreme capsules, and certain hitherto undescribed corticotegmental tracts and vertically oriented intercortical association paths of the frontal cortex and commissural pathways are considered.

\section{LITERATURE CITED}

Atules, W. F. 1923 Origin and destination of secondary visceral fibers in the guinea pig. J. Comp. Neur., \$5: $275-311$.

ANAND, S. K., AND S. DUA 1956 Circulatory and respiratory changes induced by electrical stimulation of limbic system. J. Neurophysiol., 19: 393-400.

Ariëns Kappers, C. U., G. C. Huber aNd E. C. Crosbr 1936 The Comparative Anatomy of the Nervous System of Vertebrates Including Man. Macmillan Co., New York City. Vol. II, 1845.

Bailey, P., G. Von Bonin, H. W. Garol and W. S. McCulloch 1943 Long association fibers in cerebral hemispheres of monkey and chimpanzee. J. Neurophysiol., $6: 129-134$.

Crosiy, E. C. 1956 The role of the midbrain as a part of the discharge path from higher centers. Presented at Proceedings of First International Meeting of Neurobiologists, 1956: 217-231.

Crosbr, E. C., AND R. T. Woodburne 1951 The mammalian midbrain and isthmus regions. Part II. The fiber connections. C. The hypothalamo-tegmental pathways. J. Comp. Neur., 94: 1-32. 
(rovcr, R. L. 1940 The efferent fibers of the thalamus of Macacus rhesus. Ibid., 7\%: 177-186.

Croveh, R. L., AND J. K. Thompson 1938 The efferent fibers of the thalamus of Macacus rhesus. Ibid., 69 : 225-271.

Fleming, J. F. R., And E. C. Crosby 1955 The parietal lobe as an additional motor area: The motor effeets of electrical stimulation and ablation of cortical areas 5 and 7 in monkeys. Ibid., 103: 485-512.

FreEMAN, W., AND J. W. WATTS 1947 Retrograde degeneration of the thalamus following prefrontal lobotomy. Ibid., $86: 65-93$.

Frefman, W., and J. M. Williams 1951 Lesions of transorbital lobotony. Arch. Neur. Psych., 66: 191-198.

Fulton, J. F. 1949 Physiology of the Nervous System. 3rd Ed., Oxford University Press, New York, 667.

Fulton, J. F., C. F. JACOBSEN AND M. A. Kennard 1932 A note concerning the relation of the frontal lobes to posture and forced grasping in monkeys. Brain, 55: 524-535.

Furstenderg, A. C., $\Lambda$ ND E. C. Crosby 1948 Neuron ares of elinical significance in laryngology. Ann. Otol, Rhin. Laryng., 57 : 298-310.

Huber, G. C., ANd E. C. Crosby 1929 Somatic and visceral eonnections of the diencephalon. Arch. Neur. Psych., 22: 187-229.

Hunter, J., AND H. H. JASPER 1949 Effects of thalamie stimulation in unanesthetized animals. The arrest reaction and petit mal seizures, activation patterus and generalized convulsions. EEG Clinic. Neurophysioj., 1: $305-324$.

INGRAM, W. 1940 Hypothalamic topography and connections. Assoc. Res. Nerv. Ment. Dis., 20: 195-241.

JASPER, H. H. 1949 Diffuse projection systems: the integrative action of the thalamic reticular system. EEG Clinic. Neurophysiol., 1: 405-419.

LaURsen, A. M. 1955 An experimental study of pathways from basal ganglia. J. Comp. Neur., 10\%: 1-26.

Le Gros Clarke, W. E. 1932 The structure and connections of the thalamus. Brain, $55: 406-470$.

METILE, F. A. 1947 a Extracortical conuections of the primate frontal cerebral cortex. II. Corticofugal connections. J. Comp. Neur., $86: 119-154$.

1947b Extracortical connections of the primate frontal cerebral cortex. I. Thalamo-cortical connections. Ibid., $86: 95-118$.

Mirsky, A., M. Stein and G. Paulish 1954 The secretion of an antidiuretic substance into circulation of adrenalectomized and hypophysectomized rats exposed to noxious stimuli. Endocrinology, 55: 28--39.

Monnigr, M., and R. Fischer 1950 Contributions expérimentales a la physiolo. gie du trone cérébral chez 1'homme. II. Stimulation et coagulation des noyau latero-ventral, ventro-postéro-lateral et ventro-postéro-medial du thalamus. Helv. Physiol. Pharm. Acta, $8:$ C55-58.

Muskens, L. J. J. 1914 An anatomico-physiological study of the posterior longitudinal bundle in its relation to forced movements. Brain, 36: $352-426$. 
Niemer, W. T., AND J. Jimenez-CAstellanos 1950 Corticothalamic connections in cat as revealed by physiological neuronography. J. Comp. Neur., 93 : 101-124.

PAPEZ, J. W. 1938a Thalamic connections in a hemidecorticate dog. Ibid., 69: 103-19.

$1938 \mathrm{~b}$ Reciprocal connections of the striatum and pallidum in the brain of Pithecus. Ibid., 69:329-349.

Peele, T. L. 1944 Acute and chronic parietal lobe ablations in monkeys. J. Neurophysiol., $7: 269-286$.

PETERSEN, E., AND I. BICKERS 1952 Projection of cortical area 6 to brain stem in monkey. Ibid., 15: 85-90.

Porter, R. 1954 The central nervous system and stress induced eosinopenia. Rec. Prog. Horm. Res, 10: 1-27.

Powell, T. P. S., AND W. M. CowaN 1956 A study of thalamo-striate relations in the monkey. Brain, 79: 364-391.

Pribram, K. H., ANd M. Bagshaw 1953 Further analysis of the temporal lobe syndrome utilizing frontotemporal ablations. J. Comp. Neur., 99: 347-375.

Pribram, K. H., K. L. Chow and J. Semmes 1953 Limit and organization of the cortical projection from medial thalamic nucleus in the monkey. Ibid., 98: $433-448$.

Ranson, S. W. 1940 Regulation of body temperature. Assoc. Res. Nerv. Ment. Dis., 20: 342-399.

Ranson, S. W., S. W. RANson, JR. AND M. RANson 1941 Fiber connections of the corpus striatum as seen in Marchi preparations. Arch. Neur. Psych., 46: $230-249$.

Rrocf, D. McK. 1929 Studies of diencephalon of carnivora. Part I. J. Comp. Neur., 49: 1-121.

Roberts, S., AND M. Kehlar 1956 Influence of opinephrine and cortisone on the metabolism of the hypophysis and hypothalamus of the rat. Endocrinology, $57: 64-69$.

SACHS, E. 1909 On the structure and functional relations of the optic thalamus. Brain, 3\% : 95-186.

ScharRER, E. 1954 Hormones produced by neurosecretory cells. Rec. Prog. Horm. Res, 10: 183-226.

Schneider, R. C., AND E. C. Crosby 1954 Stimulation of "second" motor areas in the macaque temporal lobe. Neurol, $4: 612-622$.

SHEPs, J. G. 1945 The nuclear configuration and cortical connections of the human thalamus. J. Comp. Neur., 83: 1-56.

Smiтh, M. 1956a Observations on the extended use of the Marchi method. J. Neurol. Neurosurg. Psych., 19: 67-74.

$1956 \mathrm{~b}$ The recognition and prevention of artifacts of the Marchi method. Ibid., 19: 74.

SPIEgeL, E. A., AND J. T. Wyois 1952 Thalamotomy and pallidotomy for treatment of choreic movements. Acta Neurochir., 2: 417-482.

STARZL, T. E., AND D. G. Whithock 1952 Diffuse thalamic projection system in monkey. T. Neurophysiol., $15: 449-467$. 
SWANK, R. L., AND H. A. DAVenport 1935 Chlorate-osmie-formalin method for staining degenerating myelin. Stain Tech., 10: 87-90.

Thiele, F. H. 1905 On the efferent relationship of the optic thalamus and Dieters' nucleus to the spinal cord with special reference to the cerebellar influx theory (Hughlings Jackson) and genesis of decerebrate rigidity (Sherrington). S. B. Roy. Soc. Proc., 76:360-365.

Travis, A. M. 1955a Neurological deficiencies after ablation of precentral motor area in Macaca mulatta. Brain, 78: 155-174.

1955b Neurological deficiencies following supplementary motor area lesions in Macaca mulatta. Ibid., 78: 174-198.

Turner, E. A. 1954 Cerebral control of respiration. Ibid., 77: 448-496.

WALKer, A. E, 1938 The Primate Thalamus. University of Chicago Press. 138.

1940 A eytoarchitectural study of the prefrontal area of the macaque monkey. J. Comp. Neur., $7 S: 59-86$.

Whitrock, D. G., AND W. J. H. NAUTA 1956 Subcortical projections from the temporal neocortex in Macaca mulatta. Ibid., 106: 185-212.

Woodburne, R. T., E. C. Crosbi ANd R. MoCoter 1946 The mammalian midbrain and isthmus regions. II. A. The relations of the tegmentum of the midbrain with the basal ganglia in Macaca mulatta. Ibid., 85: $67-92$. 


\section{PLATES}

\section{Abbreviations used in figures}

$A N G$, anterior nuclear group

$\mathrm{CE}$, extreme capsule (capsula extrema)

$\mathrm{CN}$, caudate nucleus (nucleus caudatus)

DPS, diencephalic periventrieular

system

EML, external medullary lamina

ET, electrode tract

F, fornix

FR, retroflexed fasciculus (fasciculus retroflexus)

GP, globus pallidus

II, hypothalamus

IC, internal capsule (capsula interna)

IF, internuclear fibers

IFC, inferior frontal convolution

TML, internal medullary lamina

ITP, inferior thalamic peduncle

LH, lateral hypothalamie area

MN, medial nucleus (nucleus medialis)

MSA, medial septal area

MTG, middle temporal gyrus
NA, accumbens nucleus (nucleus accumbens)

NP, paraventricular nucleus of the hypothalamus (nueleus paraventricularis hypothalami)

NLD, lateral dorsal nucleus (nucleus lateralis dorsalis)

NVL, lateral ventral nucleus (nucleus ventralis lateralis)

NVP, posterior ventral nucleus (nucleus ventralis posterior)

PHT, pallidohypothalamic tract (tractus pallidohypothalamicus)

$\mathrm{SB}$, subcallosal bundle

SI, innominate substance (substantia innominata)

SM, medullary stria (stria medullaris)

STG, superior temporal gyrus

TF, thalamic fasciculus (fasciculus thalamicus)

TO, olfactory tubercle (tuberculum of factorium) 


\section{PLATE 1}

\section{EXPLANATION OF FIGURE}

1 Parts A through $\mathrm{D}$, and $\mathrm{G}$ are taken from $16 \mathrm{~mm}$ positive movie film. Parts $E$ and $F$ are reproduced from snapshots. A-1 illustrates the position of the leg before stimulation, and A-2 after stimulation, of the medial thalamie nucleus. B-1 illustrates the position of the arm before stimulation, and B-2 after stimulation, of the medial thalamic nucleus. C-1 illustrates the expression of the face before stimulation, and C-2 after stimulation of the medial thalamic nucleus. D-1 illustrates the positions of the upper and lower extremities before stimulation, and D-2 after stimulation, of the medial thalamic nucleus in a monkey following hemidecortication. $E$ and $F$ demonstrate the apathy and indifference in animals 8 and 10, respectively, following ablation of the medial thalamic nueleus. G shows cerea flexibilitas and piloerection in animal 6. This posture was sustained for 5 minutes. 

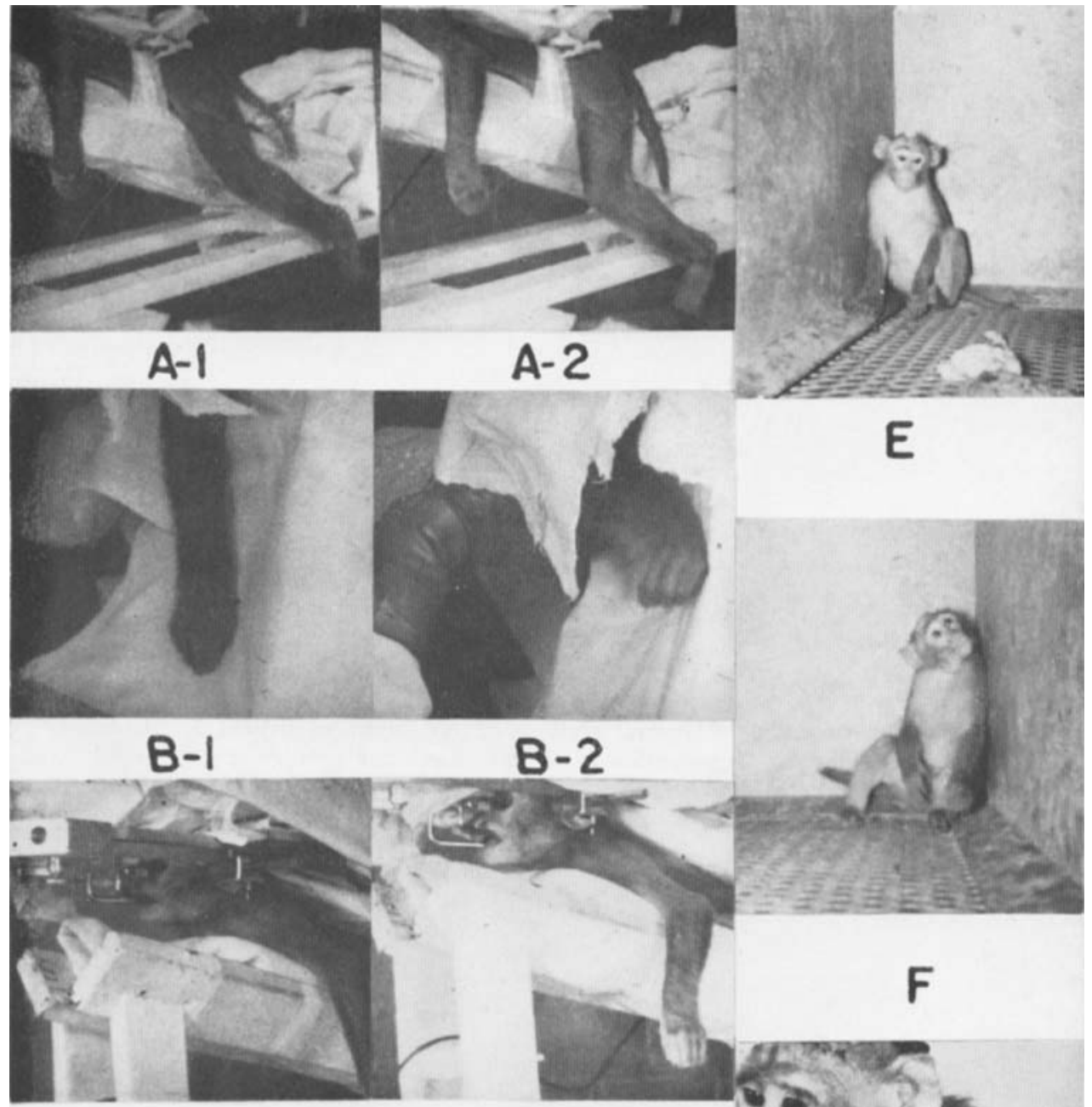

B-2
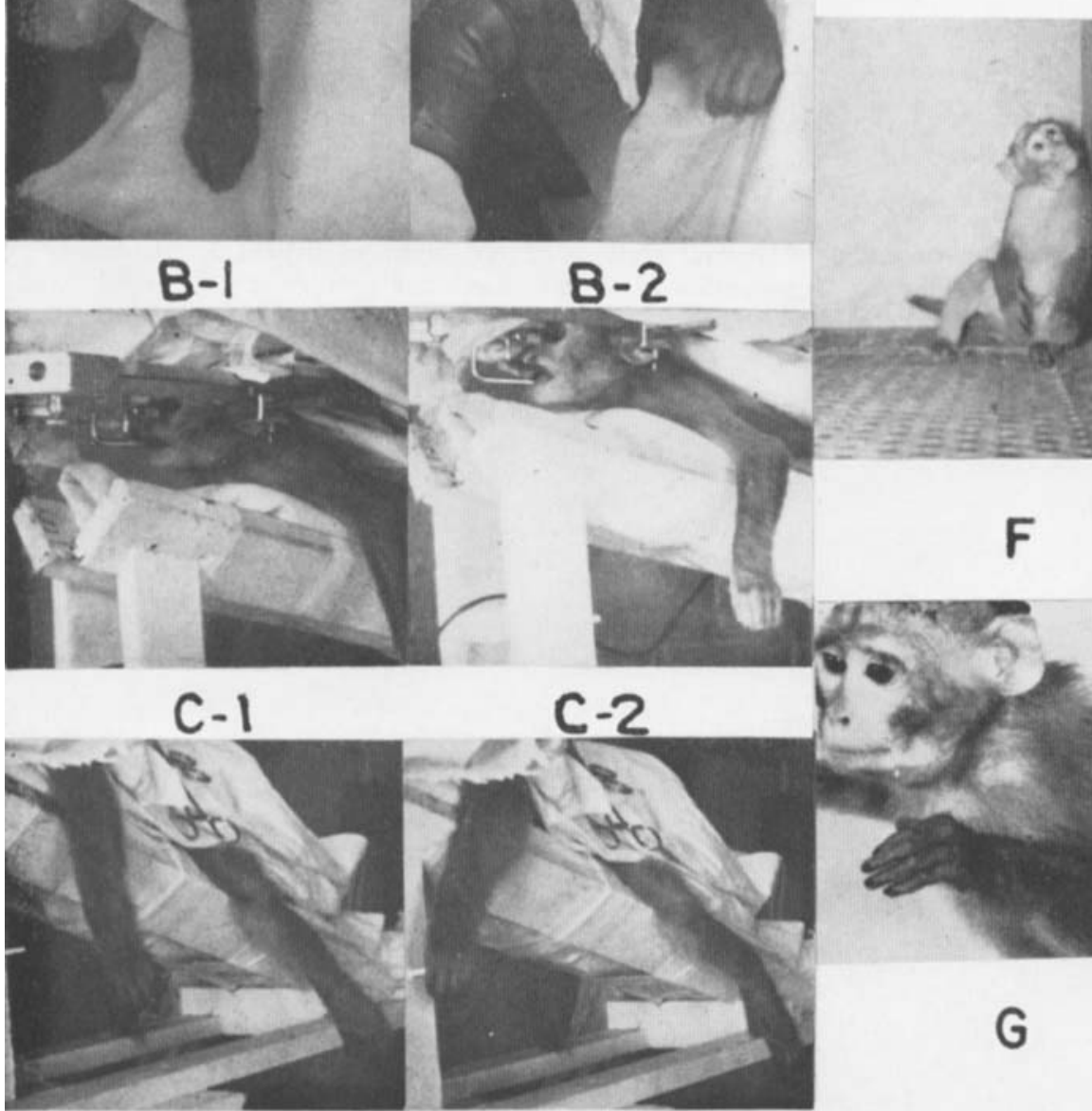
D-I
D-2 


\section{PLATE 2}

EXPLANATION OF FIGURE

2 Marehi preparation of frontal sections of the brain of animal 5. Photomicrographs show degeneration in the magnocellular portion of the medial nucleus of the dorsal thalamus $(\times 13.3)$. A is taken from the midtle region of the nucleus; $B$ from the rostral region; and $C$ from the caudal portion of the nucleus. 


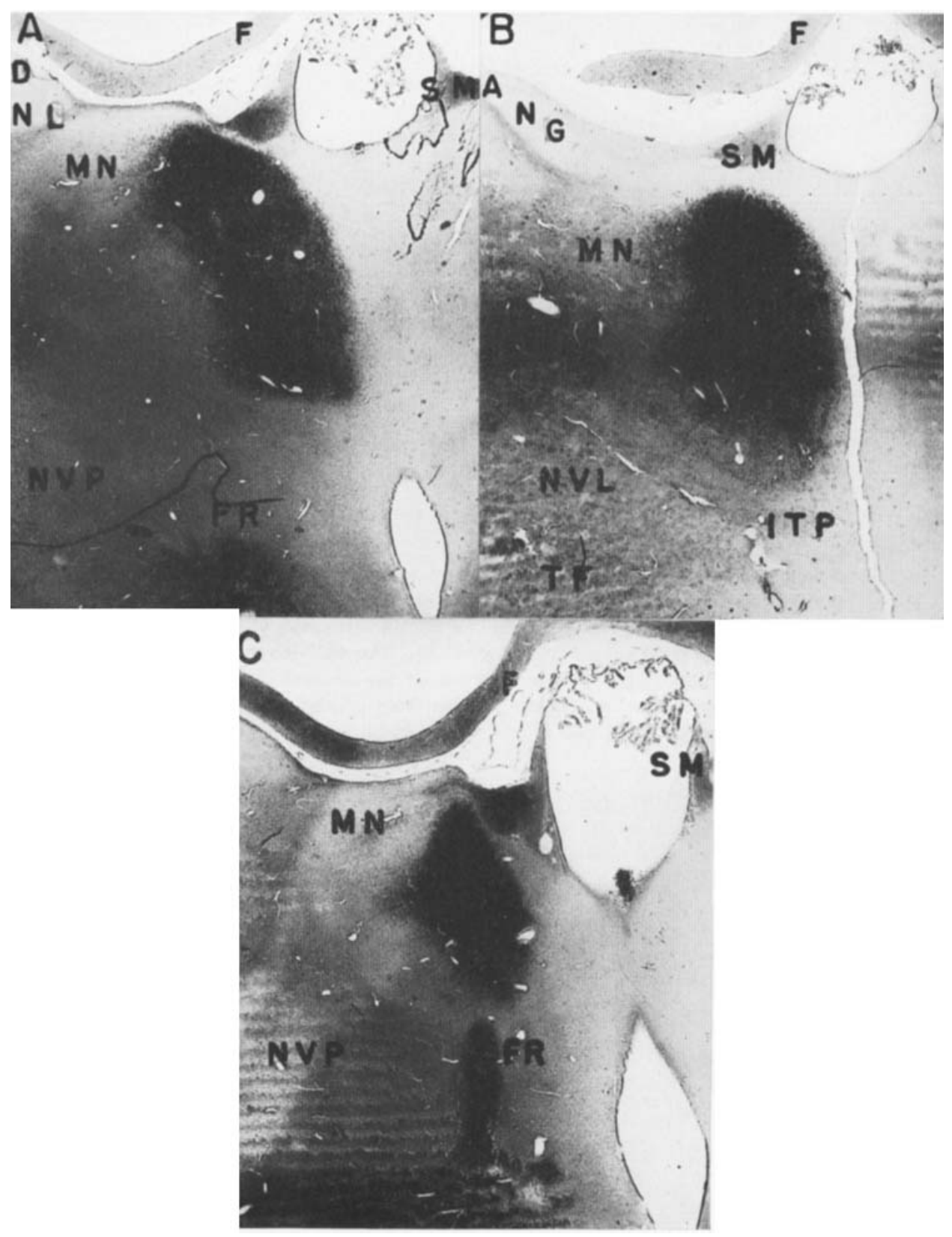




\section{PLATE 3}

EXPLANATION OF FIGURE

3 A is a photomierograph of a Marchi preparation of a frontal section through the diencephalon of animal 1 showing an clectrode tract in the medial nucleus of the dorsal thalamus $(\times 13.3)$. B is a photomicrograph of the hypothalamus of animal 7. Marchi preparation ( $\times$ 13.3). The pallidohypothalamic tract is degenerated bilaterally. $C$ is a diagram of the frontal section of the brain of the macaque. Blocks indicate the positions of the photomierographic enlargements for figures 4 and 5 . 


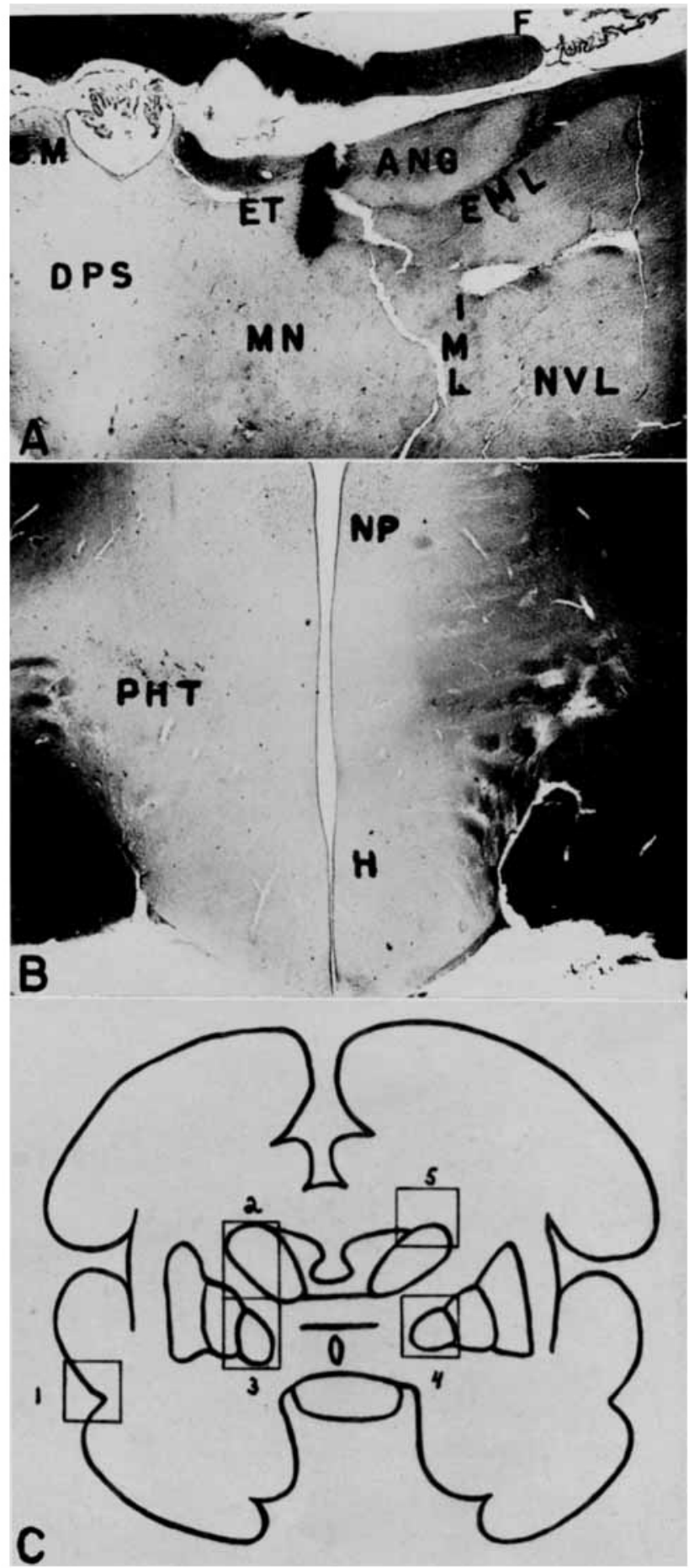


PLATE 4

EXPLANA'TION OF FIGURE

4 A represents a photomicrographic enlargment of block 1 in figure 3C. Degenerated fascicles following capsula extrema into the superior temporal gyrus are present in this Marchi preparation from the brain of animal $5(\times 13.3)$. $B$ is a photomicrographic enlargement of the internuclear striatal comnections from the Marchi preparation for animal $5(\times 13.3)$. The position of the photograph is block 2 in figure $3 \mathrm{C}$. C shows the photomicrographic enlargement of the internuclear striatal connections continued from figure $4 \mathrm{~B}$ into globus pallidus in animal 5 ( $\times 13.3)$. This is block 3 in figure $3 \mathrm{C}$. 


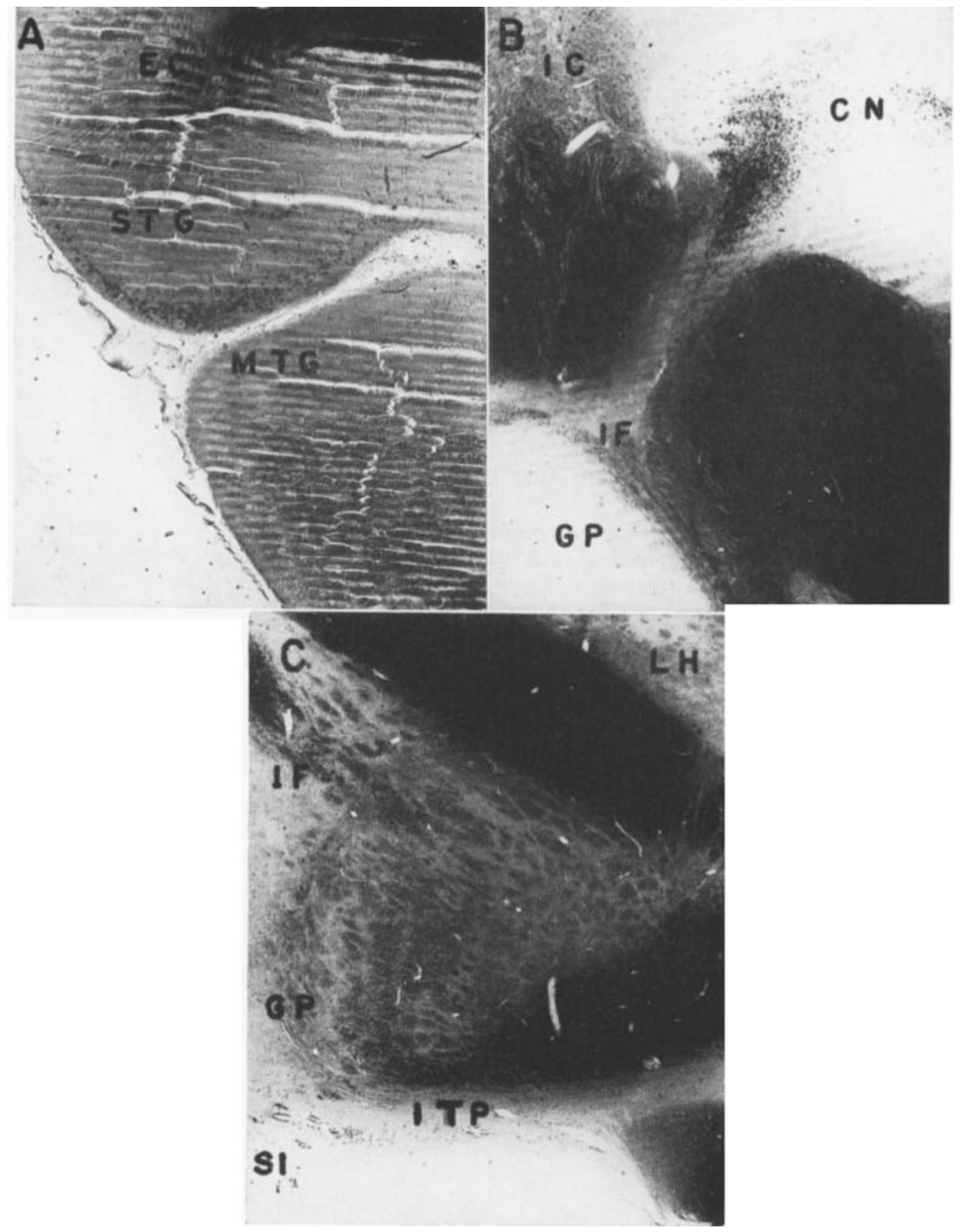




\section{PLATE 5}

EXPLANATION OF FIGURE

5 Photomicrograph slowing electrode tracts in globus pallidus ( $X$ 13.3). Marchi preparation from animal 7 . The region from which the photograph was taken is shown in block 4, figure 3C. E. Photomicrograph showing degenerating fibers at a entering the caudate nucleus through the subcallosal bundle and at $b$ through the internal capsule $(\times 13.3)$. Marchi preparation from the brain of animal 7 . Position of the section is block 5 in figure $3 \mathrm{C} . \mathrm{A}, \mathrm{B}, \mathrm{C}$ are diagrams of the surfaces of the brain of a macaque in which WalkerBrodmann areas are indicated: $A$, left lateral view; $B$, inferior frontal surface; C, medial frontal surface (Walker, '38, '40). 

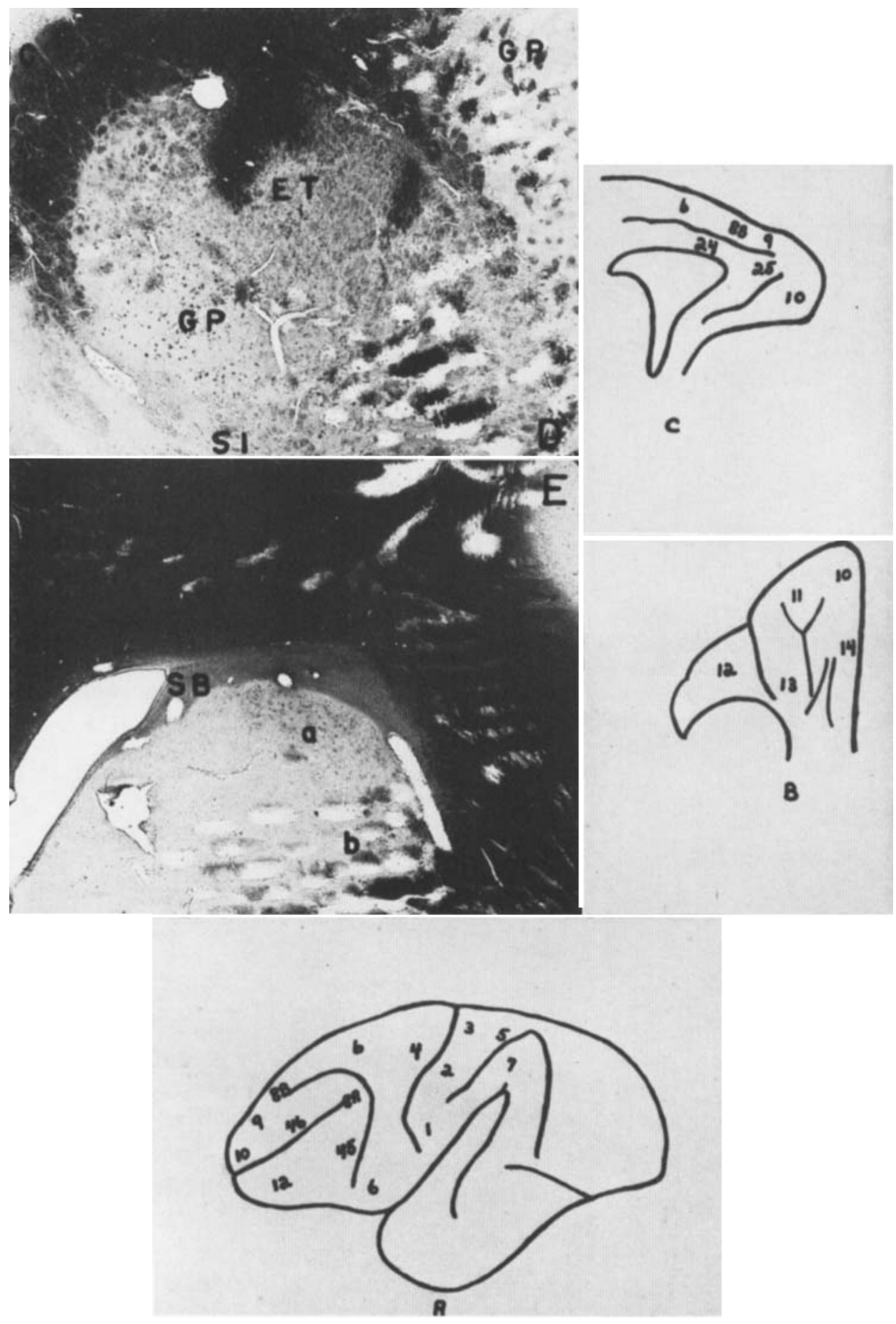
PLATE 6

EXPLANATION OF FIGURE

6 A. Lateral view of the brain from animal 6. $\mathrm{B}$ is a diagram of a frontal section through the brain of the macaque showing the position of the photomicrograph. C, photomicrographic enlargement of the degeneration in the nucleus accumbens of animal 7 . Marehi preparation $(\times 13.3)$. 

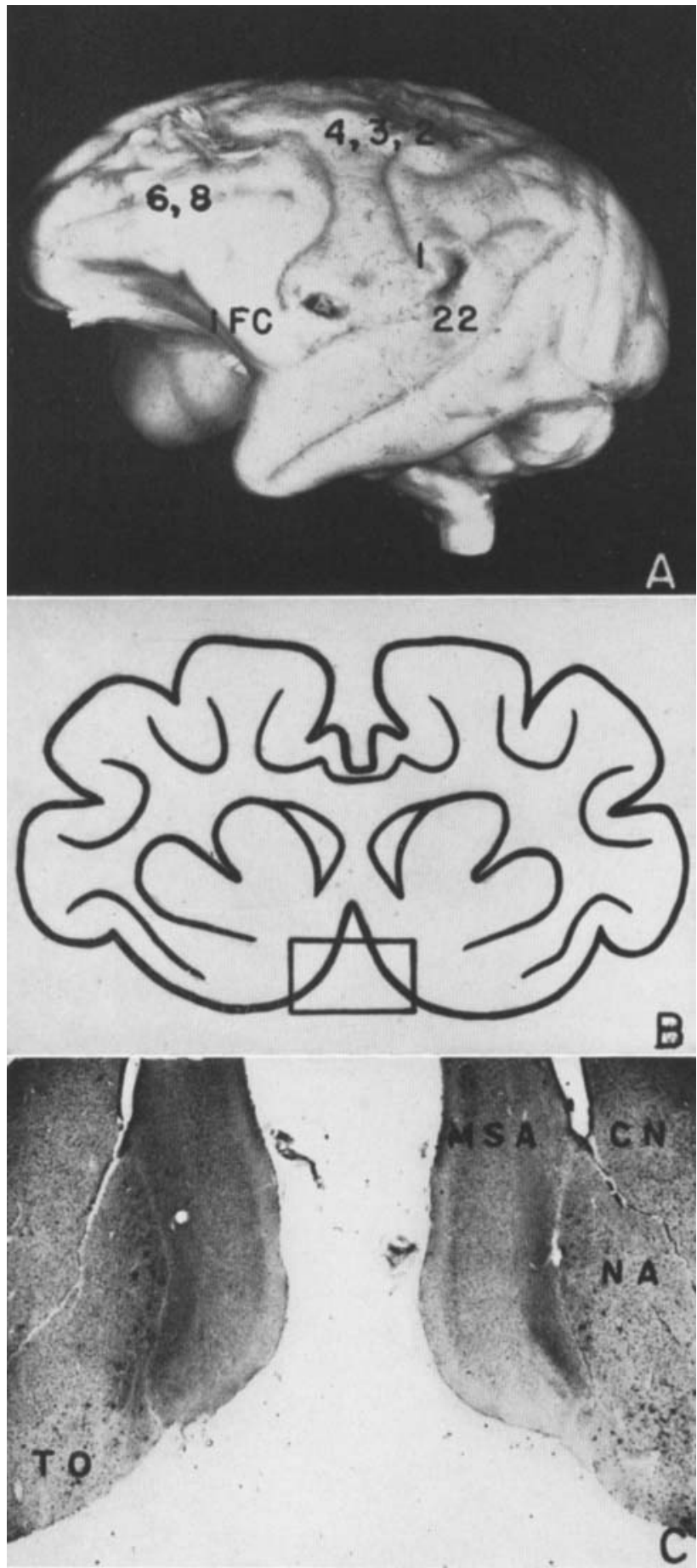